\title{
Slicing Cuts on Food Materials Using Robotic-Controlled Razor Blade
}

\author{
Debao Zhou ${ }^{1}$ and Gary McMurray ${ }^{2}$ \\ ${ }^{1}$ Department of Mechanical and Industrial Engineering, University of Minnesota, Duluth, MN 55812, USA \\ ${ }^{2}$ Food Processing Technology Division, ATAS Lab, Georgia Tech Research Institute, Atlanta, GA 30332, USA
}

Correspondence should be addressed to Debao Zhou, dzhou@d.umn.edu

Received 27 May 2011; Revised 10 August 2011; Accepted 17 August 2011

Academic Editor: Xiaosheng Gao

Copyright (C) 2011 D. Zhou and G. McMurray. This is an open access article distributed under the Creative Commons Attribution License, which permits unrestricted use, distribution, and reproduction in any medium, provided the original work is properly cited.

Cutting operations using blades can arise in a number of industries, for example, food processing industry, in which cheese, fruit and vegetable, even meat, are involved. Certain questions will rise during these works, such as "why pressing-and-slicing cuts use less force than pressing-only cuts" and "how is the influence of the blade cutting-edge on force". To answer these questions, this research developed a mathematical expression of the cutting stress tensor. Based on the analysis of the stress tensor on the contact surface, the influence of the blade edge-shape and slicing angle on the resultant cutting force were formulated and discussed. These formulations were further verified using experimental results by robotic cutting of potatoes. Through studying the change of the cutting force, the optimal slicing angle can be obtained in terms of maximum feeding distance and minimum cutting force. Based on the blade sharpness properties and the specific materials, the required cutting force can be predicted. These formulation and experimental results explained the basic theory of blade cutting fracture and further provided the support to optimize the cutting mechanism design and to develop the force control algorithms for the automation of blade cutting operations.

\section{Introduction}

Food cutting, such as potato or cheese cuts, is different from metal cuts because of the material deformability and shapevariability. Cutting mechanics formulation is constantly a hot research topic since it can provide useful information for cutting operations. In the literature, mainly two methods have been documented, that is, energy formulation method and stress tensor distribution method.

Metal cutting creates plastically deformed offcuts which permanently store energy, while the food offcuts permanently store almost no energy. Thus, many researchers have formulated the food cutting problems using energy method, most notably, using the fracture toughness concept $[1,2]$. Using energy concepts, Atkins et al. $[3,4]$ have been able to explain why the cutting fracture requires smaller force when pressing and slicing compared to pressing only. Kamyab et al. [5] formulated the stress and force distribution in cheese cutting.
Stress distribution formulation can provide an alternative explanation. Two methods can be applied: potential function method and superposition method. In the potential function method $[6,7]$, the strain and stress tensor are expressed in terms of the space derivatives of certain airy functions in the form of biharmonic equations. Using this method, the closed form expression of the stress distribution generated by a point force acting in either normal or tangential direction to the boundary of the semi-infinite body was derived in [8], the solution to a load applied normal to an infinite half-space was given by $[9,10]$, and the stress distribution generated by a tangential force applied to a surface was referred as Cerruti problems, and its solution was obtained using reciprocal theorem as summarized in [11-13]. Based on the derivation of the integral of the point force airy functions, Love [14] provided the integral for a rectangular area with constant normal pressure. Using the same method, the solution for first-order polynomial load applied to a rectangular surface has been completed by [15], 
to a triangular region has also been given by $[16,17]$, and to a circular area by [18]. The second method is to formulate the internal stress distribution, for example, using Hertz contact mechanics [19]. Yoshihara and Matsumoto [20] studied the shearing properties of wood using the stress analysis method. Lucas used fracture mechanics to explain the function of teeth in food cutting based on the detailed investigation of dental structure [21].

Blade sharpness is another factor that affects the cutting forces. Contact between the cutting object and the blade is an area, which can be shown from the microstructure of a knife [22]. Blade sharpness also directly influences the cutting moments and the grip forces applied by an operator such as the research performed by McGorry et al. [23]. Szabo et al. developed a procedure to establish knife-steeling schedules based on increased force due to knife dullness from repetitive use to minimize operator exertions and physical stress associated with work-related musculoskeletal disorders [24].

In this paper, mainly applied to the hard and crispy materials such as cheeses and potatoes, we will focus on using shear stresses at fracture to describe the cutting mechanism and blade sharpness. Firstly, the distribution of the external cutting force on the cutting-contact area is described in Section 2.1. From Sections 2.2 to 2.3, cutting force influence factors, such as the shape of the blade cutting edge, slicing angle, and so forth, are discussed. In Section 2.4, the relative sharpness factor concept built in [25] is generalized to be applicable to the pressing and slicing cuts. In Section 3, computation results are provided to illustrate the stress distribution during cutting. The computation results provide several purposes: (1) to show the stress distribution and to verify the analytical model; (2) to find out where the maximum stress is and to use them to evaluate the fracture location; (3) to understand the influence of the blade shape (cutting force shape) and slicing angle to the fracture force; (4) to figure out the fracture mode of blade cuts. Experimental results were provided in Section 4 to verify the formulation. Justification about the application of this algorithm on nonlinear materials are provided in Section 5. Conclusions were drawn in Section 6.

\section{Modeling of the Cutting Interaction}

2.1. Simplified Model of Pressing and Slicing Cuts on HardCrispy Materials. The illustration of potato cutting is shown in Figure 1. The modeling and experiments will focus on using the blade shown in Figure 1(a) to realize the robotcontrolled cuts shown in Figure 1(b). The microstructure photo of the edge of a blade is shown in Figure 2(a) with the measured width of the blade cutting edge. For that particular razor blade, the edge of this blade is $850 \mathrm{~nm}$ with flat surface. The range of the edge width of a brand new blade is around $500 \mathrm{~nm}$ to $1250 \mathrm{~nm}$ with the shape shown in Figure 2(b).

The microstructure of potatoes showed that the homogeneity of the particles is 500 times bigger than the blade edge width. Since the target problem is about cutting fracture, the focused problem is the stress distribution at the contact area at the moment just before the initiation of the cutting fracture.

At that moment, enough pressure has been built up beneath the blade, and the blade has a firm and solid contact with the target. Thus, the cutting interaction, such as the potato cutting shown in Figure 1, is simplified as a beltarea force acting on the surface of a semi-infinite body as illustrated in Figure 3(b), where the interaction between the blade and the target (Figure $3(\mathrm{a})$ ) is simplified as a beltarea force, and the target is simplified as a semi-infinite body. Given the contact length between the blade and cutting material is $l$ and the width of the blade edge is $2 a$, the contact area between the blade and the material is belt-like, that is, $(-l / 2, l / 2)$ in $x$ direction and $[-a, a]$ in $y$ direction, where point $o$ in frame $o x y z$ is at the center of the belt area, oxy plane is on the contact surface, $x$-axis is the blade length direction, and $z$-axis point into the material as shown in Figure 3(b). Normally, the width of a brand new blade (2a) ranges from $500 \mathrm{~nm}$ to $1250 \mathrm{~nm}$, and the contact length is normally bigger than one centimeter, roughly $l / a>10^{4}$, that is, the contact length in $x$ direction is much bigger than that in $y$ direction. Thus, $l$ can be considered as infinite compared to $a$.

For the symbolic expression in this paper, capital $P$ is used to express a cutting force and small $p$ is used to express a pressure variable, while subscript and superscript $p$ of a variable is used to express the variable is either pointforce or point-pressure related. Subscript and superscript $n$ stands a variable in normal or $z$ direction and subscript and superscript $t$ stands a variable in tangential or $x$ direction. For example, $P_{p}$ is an ideal point force acting on the contact surface of a semi-infinite body with normal component $P_{p n}$ and tangential component $P_{p t}$, while $p_{n}$ and $p_{t}$ are the cutting pressure components in the $z$ direction and $x$ direction, respectively. Subscript and superscript $l$ is used to express the variable is line-pressure related.

Force $P$ has two components: normal force $P_{n}$ and tangential force $P_{t}$ as shown in Figure 4 . Then there are

$$
P_{n}=P \cos \alpha, \quad P_{t}=P \sin \alpha,
$$

where $\alpha$ is defined as the slicing angle. If we assume the pressure does not change along a line parallel to the $x$-axis, the line-pressures on a line parallel to $y$-axis are $p_{l n}$ in normal direction and $p_{l t}$ in tangential direction, there are

$$
P_{n}=p_{l n} l, \quad P_{t}=p_{l t} l .
$$

The area pressures $p_{n}$ and $p_{t}$ can be used to calculate line pressures $p_{l n}$ and $p_{l t}$ and total force $P_{n}$ and $P_{t}$ as follows:

$$
\begin{array}{cc}
P_{n}=\int_{-l / 2}^{l / 2} \int_{-a}^{a} p_{n} d s d v, & P_{t}=\int_{-l / 2}^{l / 2} \int_{-a}^{a} p_{t} d s d v, \\
p_{l n}=\int_{-a}^{a} p_{n} d v, & p_{l t}=\int_{-a}^{a} p_{t} d v .
\end{array}
$$

Since the dimension of the blade edge is around $1000 \mathrm{~nm}$ ( $1 \mathrm{um}$ ), there is no force sensor in such small size to measure the stress distribution. According to the edge shape of a razor 


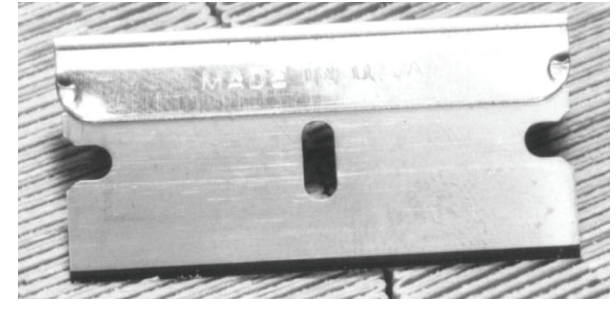

(a) Razor blade used in this research

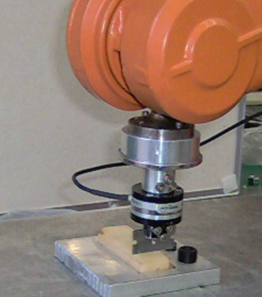

(b) Robotic potato cut

Figure 1: Razor blade cutting.

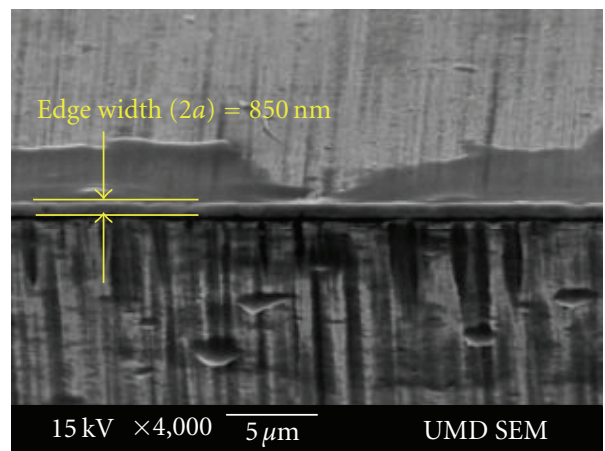

(a) Microstructure of a blade cutting edge

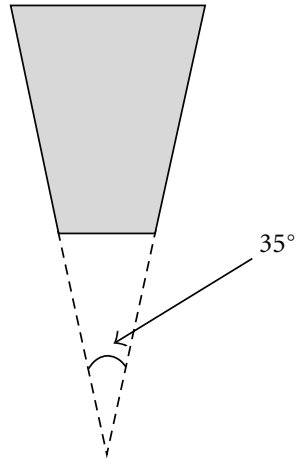

(b) Shape of a blade cutting edge

FiguRE 2: Microstructure of the edge of the razor blades used in the modeling and experiments.

blade, the cutting force distributions in both tangential and normal direction to the cutting surface have to be assumed. In this paper, they are assumed to be trapezoid as shown in Figure 5 and their mathematical expressions are summarized in Table 1.

There are

$$
\begin{gathered}
p_{l n}=\int_{-a}^{a} p_{n} d v=q_{n}(a+w), \\
p_{l t}=\int_{-a}^{a} p_{t} d v=q_{t}(a+w), \\
P_{n}=P \cos \alpha=p_{l n} l=q_{n}(a+w) l, \\
P_{t}=P \sin \alpha=p_{l t} l=q_{t}(a+w) l .
\end{gathered}
$$

Then there are

$$
q_{n}=\frac{P \cos \alpha}{(a+w) l}, \quad q_{t}=\frac{P \sin \alpha}{(a+w) l} .
$$

Other simplifications in this paper are as follows. (1) Relaxation and creep are ignored since we consider the instantaneous cuts. (2) The cutting force intensity is zero at the edge of the contact area. (3) The offcuts move away from the knife and no friction force acts on the side of the blade; the contact area between the blade edge and the material does not change once full contact is established.
2.2. Modeling of the Stress Distribution in the Cutting Materials. The modeling starts from a stress field generated by a point force on a semi-infinite solid body. Superposition method is used to obtain the stress field generated by a beltshape area force on a semi-infinite solid body. The procedure is Table 2. In the last row of Table 2, the functions $f_{1}, f_{2}, f_{3}$, $f_{4}, f_{5}$, and $f_{6}$ can be explicitly expressed using (A.8) to (A.10) and (A.12) to (A.14) using the variables $y, z, a, w$. The details are shown in the appendix.

Although the modeling method uses a standard superposition which can be found in any material mechanics textbook, it is first time in the literature that the stress distribution due to a belt-shaped area force was obtained in a closed and manageable form.

Substituting $q_{n}$ and $q_{t}$ in (5) into $(*)$ in Table 2, the stress tensor at point $A(x, y, z)$ generated by cutting force $P$ is obtained as

$$
\begin{aligned}
{[\sigma] } & =\left[\begin{array}{ccc}
\sigma_{x} & \tau_{x y} & \tau_{x z} \\
\tau_{x y} & \sigma_{y} & \tau_{y z} \\
\tau_{x z} & \tau_{y z} & \sigma_{z}
\end{array}\right] \\
& =\frac{P}{l(a+w)}\left[\begin{array}{lll}
f_{1} \cos \alpha & f_{4} \sin \alpha & f_{5} \sin \alpha \\
f_{4} \sin \alpha & f_{2} \cos \alpha & f_{6} \cos \alpha \\
f_{5} \sin \alpha & f_{6} \cos \alpha & f_{3} \cos \alpha
\end{array}\right] .
\end{aligned}
$$




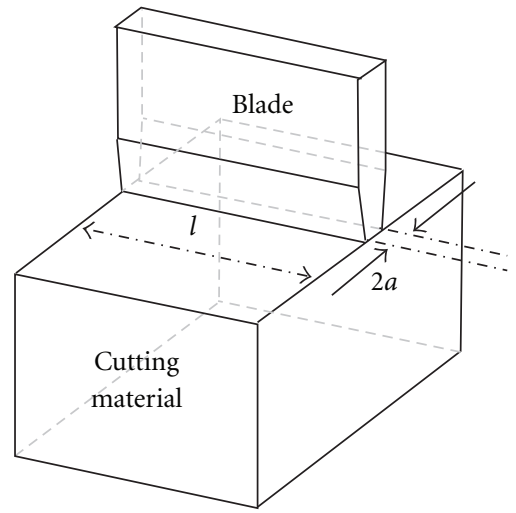

(a) Illustration of the cut

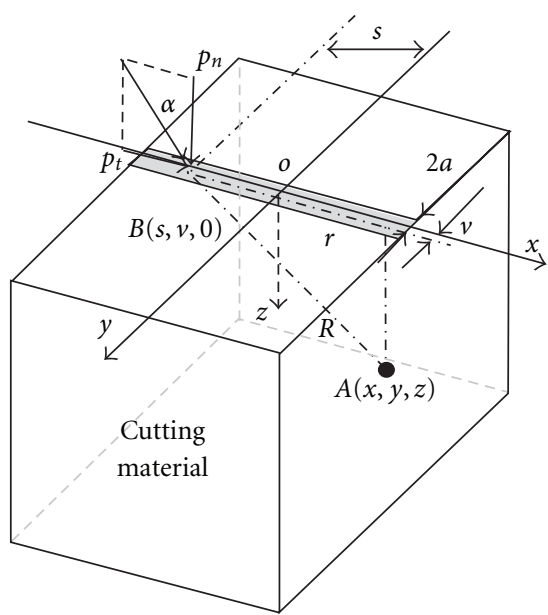

(b) Replacing the blade with belt-shape area force

FIGURE 3: Simplified cutting model.

The principle stresses $\sigma_{1}, \sigma_{2}, \sigma_{3}$ can then be obtained through solving

$$
[\sigma] \mathbf{x}=\lambda \mathbf{x},
$$

where the solutions to $\lambda$ are the principle stresses $\sigma_{1}, \sigma_{2}$, and $\sigma_{3}$; the solutions to $\mathbf{x}$ ( 3 by 1 vector) express the direction of the principle stresses. The maximum shear stress $\left(\tau_{i j}\right)$ can then be obtained as

$$
\tau_{i j}=\frac{1}{2}\left(\sigma_{i}-\sigma_{j}\right),
$$

where $i$ and $j$ are one of the numbers 1,2 , or 3 .

2.3. Fracture and Initialization during Blade Cutting. Tresca's failure criterion [26] was adopted to identify fracture initialization during blade cutting. This criterion can be expressed as

$$
\tau_{\max }=\operatorname{Max}\left(\operatorname{abs}\left(\tau_{12}\right), \operatorname{abs}\left(\tau_{23}\right), \operatorname{abs}\left(\tau_{13}\right)\right) \geq \frac{\tau_{u}}{K_{s}},
$$

where $\tau_{u}$ is the ultimate shear strength, $K_{s}$ is the ratio of the fracture initialization force over continuous cutting force, the function abs(\#) returns the absolute value of the expression \#, and the function $\operatorname{Max}(x, y, z)$ returns the biggest value among $x, y$, and $z$.

During the cutting process, there are two sets of fracture: fracture initialization and postfracture. Prior to material fracture, all of the stresses in the material are due to deformation. At the moment of fracture initialization, (9) can be applied with $K_{s}=1$. After the fracture initiation, postfracture criterion will apply. During postfracture, the initial failure criterion $\left(K_{s}=1\right)$ is no longer valid. The stress concentration factor needs be determined experimentally using

$$
K_{s}=\frac{\tau_{u}}{\tau_{o}}=\frac{P_{u}}{P_{c}}
$$

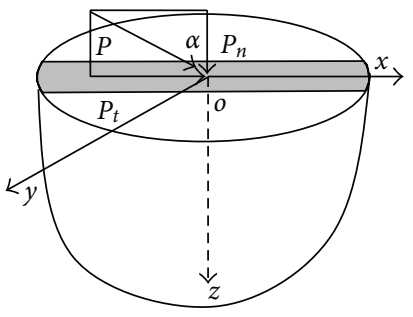

FIGURE 4: Cutting force acting on a semi-infinite body with slicing angle $\alpha$.

where $\tau_{u}$ and $\tau_{o}$ are the ultimate shear stress at fracture initialization (material property) and the ultimate stress during postfracture (determined experimentally), respectively, and $P_{c}$ is the cutting force during continuous cutting (postfracture), $P_{u}$ is the cutting force at the moment of fracture initiation. From (6)-(9), there is

$$
\tau_{i j}=\frac{P}{l(a+w)} f_{7 i j}(y, z, a, w, \alpha),
$$

where function $f_{7 i j}(\#)$ expresses a function of variable \#. $f_{7 i j}(\#)$ can be explicitly expressed using (A.8) to (A.10) and (A.12) to (A.14). At the moment of fracture initiation, the required force is obtained by substituting (11) into (9),

$$
\begin{gathered}
P_{u}=\frac{\tau_{u} l(a+w)}{K_{s} f_{8}\left(y_{u}, z_{u}, a, w, \alpha\right)}, \\
P_{n u}=P_{u} \cos \alpha, \quad P_{t u}=P_{u} \sin \alpha,
\end{gathered}
$$

where $P_{u}$ is the cutting force during cutting fracture and $\left(y_{u}, z_{u}\right)$ represents the $y$ and $z$ coordinates of the fracture location, and function $f_{8}(\#)$ can be explicitly expressed using (9) and (11). $P_{n u}$ is the normal cutting force and $P_{t u}$ is the tangential cutting force. 


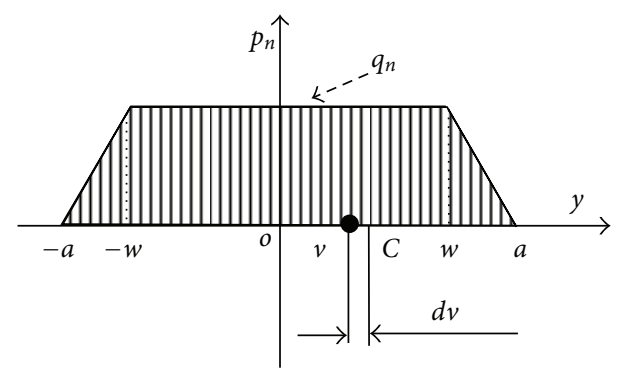

(a) Normal pressure distribution

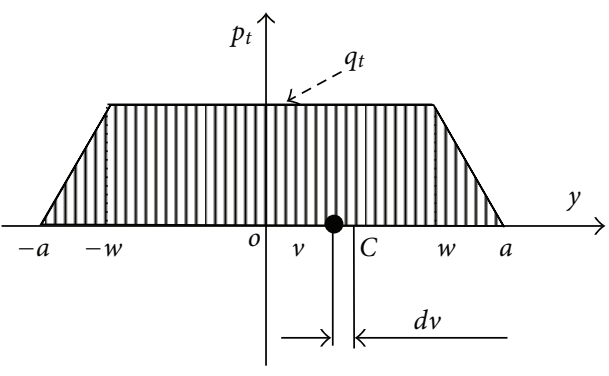

(b) Tangential pressure distribution

Figure 5: Force distribution profile along $y$-axis at $z=0$, that is on the contact surface.

TABLE 1: Pressure distribution along a line in $y$-axis direction.

\begin{tabular}{lcc}
\hline Normal direction & Tangential direction & Locations on $y$-axis \\
\hline$p_{n}=0$ & $p_{t}=0$ & $y<-a$, \\
$p_{n}=\frac{q_{n}}{a-w}(y+a)$ & $p_{t}=\frac{q_{t}}{a-w}(y+a)$ & $-a \leq y<-w$, \\
$p_{n}=q_{n}$ & $p_{t}=q_{t}$ & $-w \leq y<w$, \\
$p_{n}=\frac{q_{n}}{w-a}(y-a)$ & $p_{t}=\frac{q_{t}}{w-a}(y-a)$ & $w \leq y \leq a$, \\
$p_{n}=0$ & $p_{t}=0$ & $y>a$. \\
\hline
\end{tabular}

2.4. Shape of Blade Edge: Blade Relative Sharpness Factor. In general, "sharpness of a blade" is an approximate measurement of the magnitude of the applied force $P_{u}$ in different cuts by keeping the cutting material $\left(\tau_{u}\right)$ and cutting manners ( $l$ and $\alpha$ ) unchanged. It may be possible to explicitly express the relationship between the cutting force and the factors, $a$, $w, l, \alpha$, and $\left(y_{u}, z_{u}\right)$ using (12). However, since the magnitude of $w$ and $a$ is in several hundred nanometers, it will not be practical to put a microscope in a workshop to measure the width for each blade in order to estimate its sharpness. So a relative sharpness factor is defined as follows:

$$
\eta=\frac{(a+w)}{K_{s} f_{8}\left(y_{u}, z_{u}, a, w, \alpha\right)} .
$$

When setting $K_{u}=l \tau_{u}$, there is

$$
P_{u}=l \tau_{u} \eta=K_{u} \eta .
$$

Let $P_{u 0}$ be the cutting force of the sharpest knife, $P_{u f}$ be the cutting force of the dullest knife, and $P_{u}$ be any other cutting force. In the sharpest case, such as a brand new blade from factory, the blade relative sharpness factor is defined as

$$
\eta_{0}=\frac{P_{u 0}}{K_{u}}
$$

The relative sharpness of certain blade can be expressed as

$$
\eta=\eta_{0} \frac{P_{u}}{P_{u 0}} .
$$

In the dullest case (in which the maximum allowable force has to be applied in order to realize cutting), there is

$$
\eta_{f}=\eta_{0} \frac{P_{u f}}{P_{u 0}} .
$$

Then for convenience, another parameter, knife relative sharpness level, $\kappa$ is defined as

$$
\kappa=\operatorname{int}\left(\frac{(n-1)\left(\eta-\eta_{0}\right)}{\eta_{f}-\eta_{0}}+1\right),
$$

where $n$ is a user-defined integer which is used to distinguish the sharpness level of a blade and int(\#) is a function to round the number \# to the nearest integer. The function int(\#) forces the knife sharpness level value to an integer. Substituting (15)-(17) into (18), there is

$$
\kappa=\operatorname{int}\left(\frac{(n-1)\left(P_{u}-P_{u 0}\right)}{P_{u f}-P_{u 0}}+1\right) .
$$

It can be easily seen that the knife relative sharpness level can be determined using the cutting forces at different conditions. Since the cutting forces are measureable, a knife can be categorized into $n$-level sharpness starting from level 1 by defining $\eta_{0}=1$ in the sharpest case.

\section{Simulation Analysis}

In the computation, the change of the stresses with the parameters $l$ (contact length), $a$ (half width of the blade cutting edge), $w$ (load shape), $y$ and $z$ (different locations in material) are discussed, and the stress distributions in the material under different conditions have been visualized for further parametric study. By changing the cutting force profile, the influence from blade sharpness can be observed and the fracture modes are obtained. In the calculation, the coordinate values or distance data are normalized by $a$ (half width of the blade edge), the stresses generated by normal and tangential forces are normalized by the maximum pressure $q_{n}$ and $q_{t}$, respectively, (or set $a=1 \mathrm{~mm}, q_{n}$ $=1 \mathrm{pa}$, and $\left.q_{t}=1 \mathrm{pa}\right)$.

3.1. Stress Variation with Depth. The results shown in this section are to illustrate the change of the stress distribution with the depth $(z)$ in the material at different $y$ locations. The results were obtained using $(*)$ in Table 2 by assuming $w=$ $0.8 a$, constant pressure, $q_{n}=1 \mathrm{pa}$, and $q_{t}=1 \mathrm{pa}$. The results of the stresses $\sigma_{x}, \sigma_{y}, \sigma_{z}, \tau_{y z}, \tau_{y x}, \tau_{x z}$ have been calculated and only the results for stress $\tau_{y z}$ are shown in Figure 7. In Figure 7, each curve represents the stresses change with $z$ 
TABLe 2: Area external force.

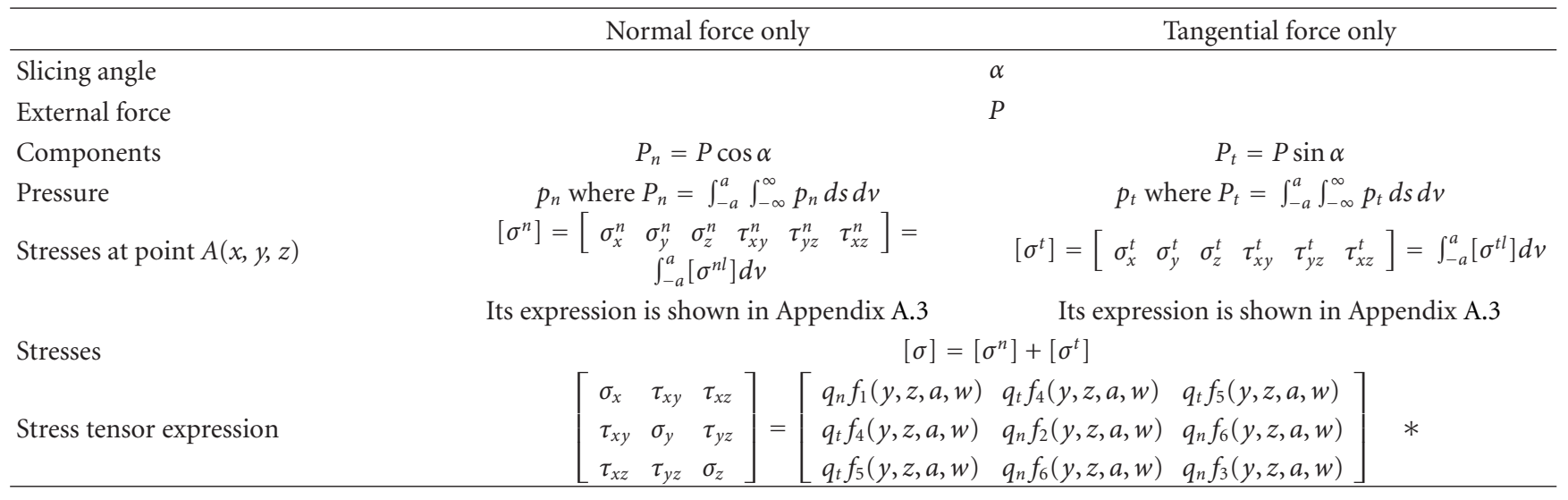

(from 0 to $3 a$ or $20 a$ ) on the same $y$ coordinates ( $y$ from $-2 a$ to $2 a)$.

It is observed from the results of $\sigma_{x}, \sigma_{y}, \sigma_{z}, \tau_{y z}, \tau_{y x}, \tau_{x z}$ that at the contact surface, the maximum stress may not exceed the cutting stress from the blade and the stress will decrease dramatically as $z$ increases. At the location where the $z$ coordinate (depth) is about twice of the half width of the cutting blade (at $z=2 a$ ), the force decreases roughly to half of the maximum stress. In general, the magnitude of the width of a sharp blade is about $1 \mathrm{um}$ and the size of the inhomogeneity in potatoes and cheese is bigger than $0.1 \mathrm{~mm}$ (100 um). This means that at the depth there are inhomogeneity, the stress already reduced to very small value.

Moreover, this paper is only interested in the stress to generate the fracture. From the calculation, it is found when slicing angle is smaller than $10^{\circ}$, the fracture happens at the depth of $z=0.37 a$ or at about $z=0.06-0.32$ um; when slicing angle is bigger than $10^{\circ}$, the fracture happens at the contact surface or at the depth of $z=0 \mathrm{um}$. Thus, in the microscale sense, the material can be considered as homogenous although in macrosense, the cutting material may be anisotropic or heterogeneous. When we only consider the stress to generate the cutting fracture, the material can be considered as homogenous.

Here we define the zone with at least tenth of the applied maximum stress from the blade as the effective zone. Since the depth of the effective zone in the cutting modeling is much smaller than the size of the fiber, or inhomogeneous, or anisotropic zone of the cutting materials, although the material itself is inhomogeneous or anisotropic, only in the cutting effective zone can the cutting modeling still be approximated as homogenous material.

\subsection{Change of the Stress Distribution with Cutting Width.} This calculation is to show the variation of the stresses with different $w$. The results are shown in Figure 8 where the abscissa is $y$ coordinate with $-2 a \leq y \leq 2 a$ and the ordinate is the stress magnitude at different $z$. In Figure 8, each curve represents the stress change with different $z$ coordinates between 0 and $a$ with step size at $0.2 a$. The $z$ values have been marked on each curve. For clarity, the different stress components are drawn with different line styles.

The subplots in Figure 8 show the stresses under three different external force distribution profiles, $w=a$ or rectangle-shaped profile is shown in Figure $8(\mathrm{a}), w=0.85 a$ or a trapezoid shaped profile is in Figure $8(\mathrm{~b})$ and $w=0$ or a triangle-shaped profile is in Figure $8(\mathrm{c})$. It is observed that all the stress components, except $\tau_{y z}$, have the maximum magnitude when $z=0$. They then decrease as $z$ increases. The maximum $\tau_{y z}$ happens inside the material at $z=0.37 a$. Since $a$ is very small (in the magnitude of $10^{-9} \mathrm{~m}$ ), the location of maximum $\tau_{y z}$ is very close to the surface. The maximum values of $\sigma_{y}, \sigma_{z}$ and $\tau_{x z}$ are in the same magnitude of $q_{n}$ or $q_{t}$, respectively.

3.3. Fracture Force via Blade Shape. The knife shape can be defined using the parameters $l, a$, and $w$. If $l$ and $a$ increase, the maximum pressure will decrease when the total external force keeps unchanged. The influence of the edge shape can be roughly expressed using $w$ (please refer to Figure 5). Using the expression in (6), the stress distributions for the different values of the $w$ on the blade-material contact plane oxy are obtained and shown in Figure 9. From Figure 9, it is observed that by keeping the external force unchanged, when the force distribution changes from constant intensity to linear intensity, the maximum magnitude of all the stresses increases. This leads to an increase in the magnitude of the maximum shear stress. Thus, by assuming $\tau_{u}$ is constant, the external force to realize cutting fracture will decrease. Or in order to realize cutting on the same material with the same constraints, the blade with the linear edge shape will use less force than any other blades. It will be the sharpest one.

3.4. Fracture Force via Slicing Angle. In this section, the knife shape and the external force intensity profile are fixed with $w=0.85 a$. The obtained stress distribution is shown in Figure 8 (b). It is observed that there are four possible locations with maximum stress, and they are summarized in Table 3 .

The changes of the corresponding stresses distribution, the principle stresses and the maximum shear stress, with 


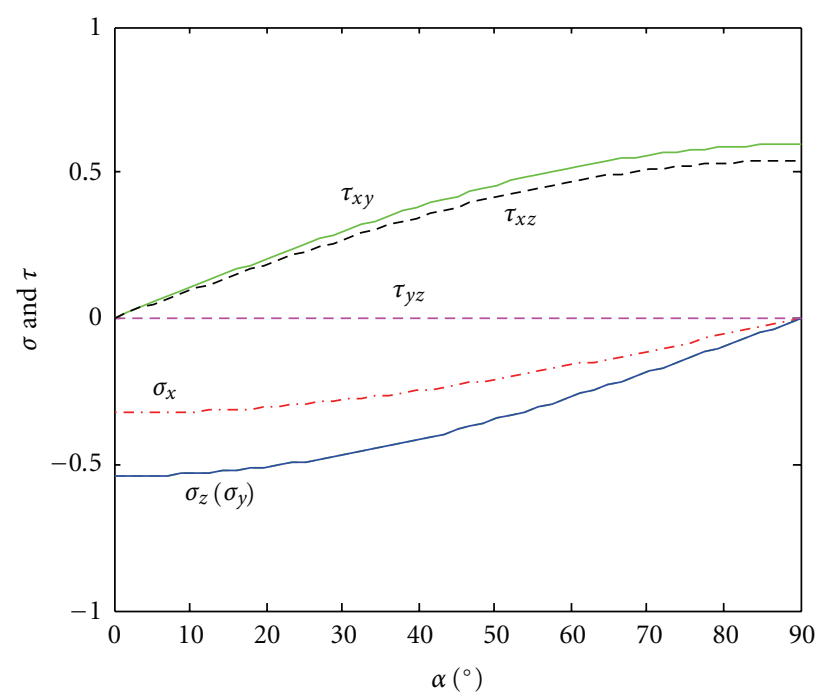

(a) Stresses

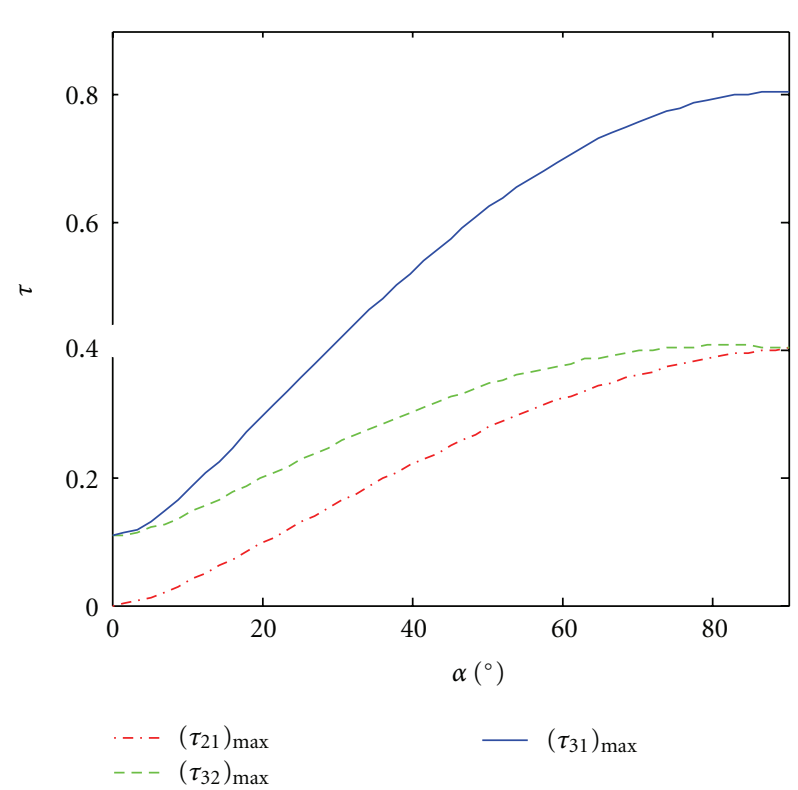

(c) Maximum shear stresses

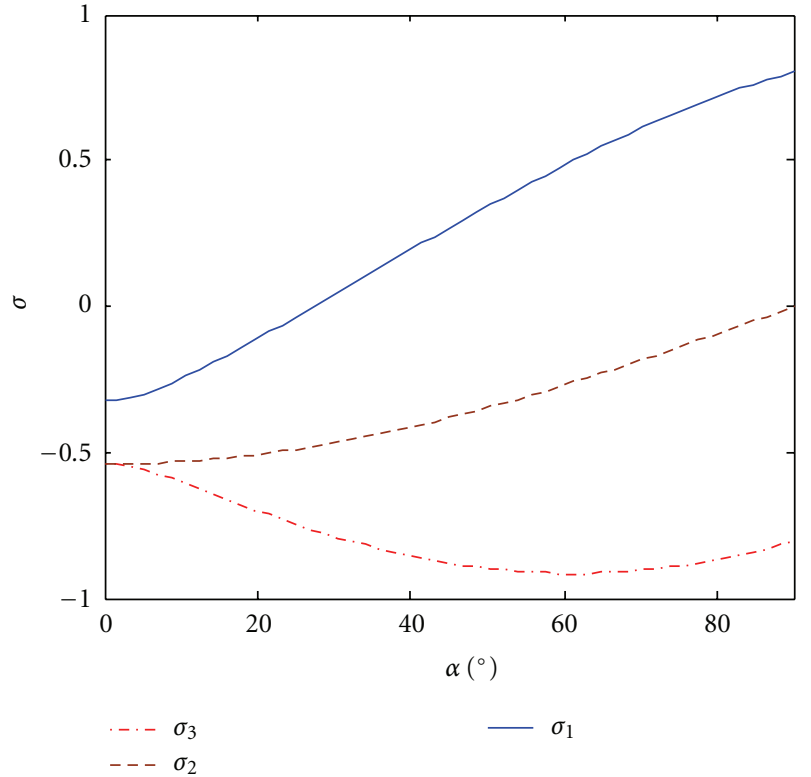

(b) Principle stresses

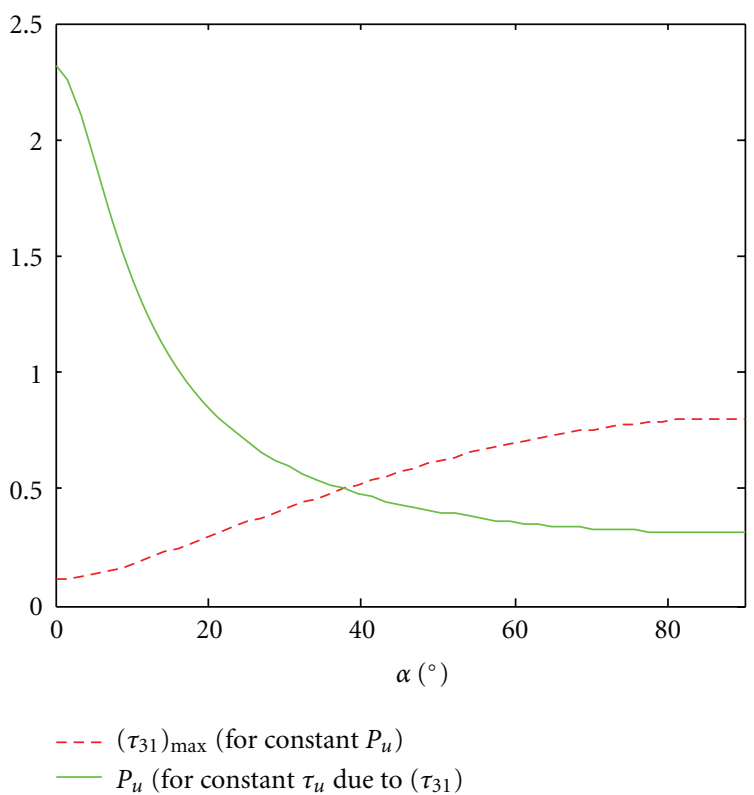

(d) Maximum shear stresses and fracture force

FIgURE 6: Stresses and external forces change with slicing angle at location (ii).

slicing angle, are shown in Figures 6(a), 6(b), and 6(c), respectively. In Figure 6, only the stresses at location (ii) $(y / a, z / a)=(-.85, .00)$ are illustrated. Similar results at locations (i), (iii), and (iv) can be obtained accordingly. The results in Figure 6(a) are obtained using (6) by assuming $P / l=1$, that is, $p_{l n}=1$ when $\alpha=0^{\circ}$ and $p_{l t}=1$ when $\alpha=90^{\circ}$. The results in Figures 6(b) and 6(c) are obtained using (7) and (8), respectively. Using Tresca's fracture criterion [26], if $\tau_{u}$ is generated by $\left(\tau_{31}\right)_{\max }$, according to (9) by setting $K_{s}=1$, the cutting force $P_{u}$ is obtained using (12) as shown in Figure 6(d). Using the same method, the maximum shear stresses and the corresponding external cutting forces at locations (i), (iii), and (iv) can also be obtained. The results are shown in Figure 10(a).

Since the external force $P_{u}$ is obtained using the same ultimate shear stress $\tau_{u}$, the smallest one among all the $P_{u}$ at the four locations from (i) to (iv) will initiate cutting fracture. For clarity, the largest maximum shear stress and its required smallest external force are redrawn in Figure 10(b). It can be observed from Figure 10(b) that when $\alpha$ is from $0^{\circ}$ to $10^{\circ}$, the shear stress at location (iv) will initialize fracture, and when $\alpha$ is from $10^{\circ}$ to $90^{\circ}$, the shear stress at location (ii) will initialize fracture. 


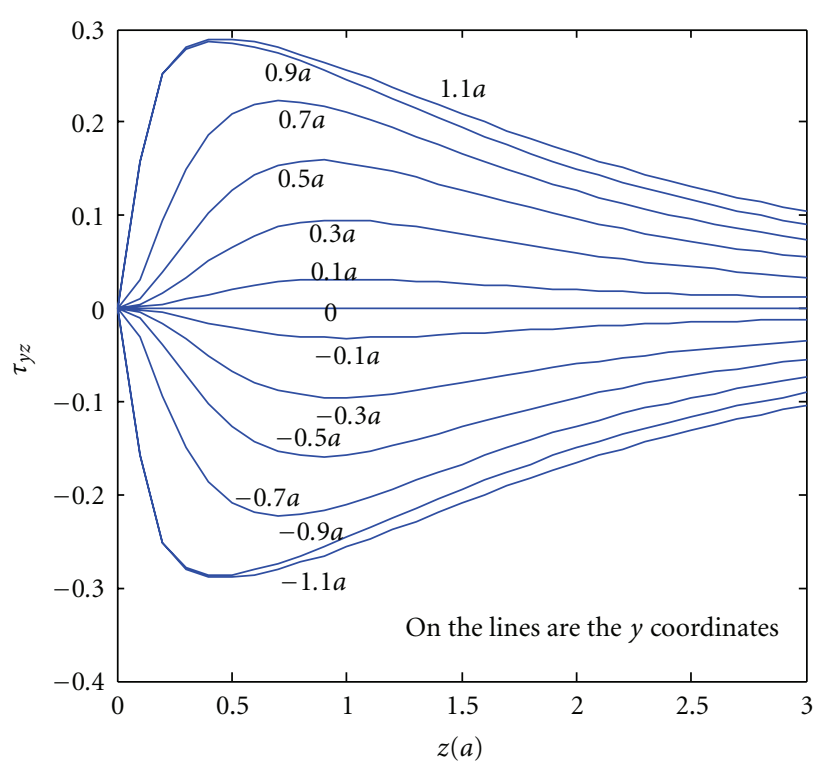

(a) $\tau_{y z}$ at different $y$ when $z<3 a$

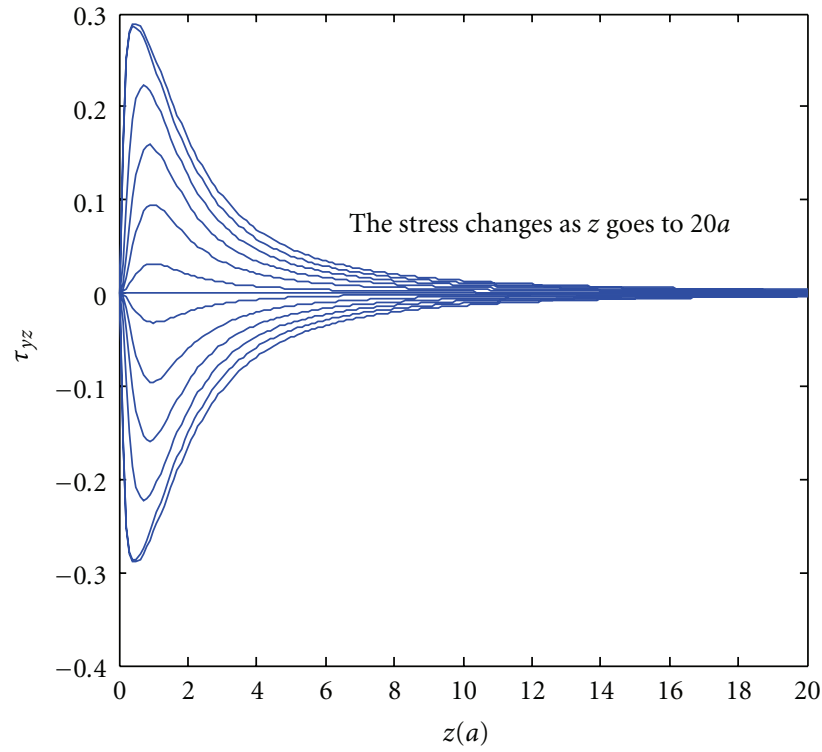

(b) $\tau_{y z}$ at different $y$ when $z<20 a$

FiguRE 7: Stress tensor changes along $z$ for different $y$.

TABLE 3: Locations and values of the possible maximum stresses.

\begin{tabular}{llllcccc}
\hline Location & $(y / a, z / a)$ & $\sigma_{x} / q_{n}$ & $\sigma_{y} / q_{n}$ & $\sigma_{z} / q_{n}$ & $\tau_{x y} / q_{t}$ & $\tau_{y z} / q_{n}$ & $\tau_{x z} / q_{t}$ \\
\hline (i) & $(.00, .00)$ & -.324 & -.541 & -.541 & 0.00 & 0.00 & .541 \\
(ii) & $(-.85, .00)$ & -.324 & -.541 & -.541 & .596 & 0.00 & .540 \\
(iii) & $(-.93, .00)$ & -.151 & -.252 & -.252 & .724 & 0.00 & .252 \\
(iv) & $(-.93, .37)$ & -.141 & -.254 & -.254 & .280 & -.163 & .234 \\
\hline
\end{tabular}

The total cutting force and its tangential and normal components are shown in Figure 11. From Figure 11, it can be seen that the fracture at slicing angle from $0^{\circ}$ to $10^{\circ}$ is due to $\tau_{y z}$ which is just beneath the surface ( $z=0.37 a$ or roughly $z \approx 370 \mathrm{~nm}$ ). By considering the stress direction, it can be seen that it is the mode II fracture, edge-sliding fracture [1]. The fracture from $10^{\circ}$ to $90^{\circ}$ is due to $\tau_{x y}$ and $\tau_{x z}$ which is just on the surface $(z=0)$. It is the mode III fracture, outof-plane tearing [1]. From the external force profile shown in Figure 11, the influence of the slicing angle can be clearly seen. The required force to cut by pressing-only is far larger than the force required by pressing-and-slicing cuts. Note also is that force $P_{t u}$ does not change a lot from $10^{\circ}$ to $90^{\circ}$ in the mode III fracture and its value is just the total force when the slicing angle is $90^{\circ}$.

\section{Experimental Verification}

Raw Russet Baking Potatoes, purchased from a local Kroger Store in Atlanta Ga, USA, were selected as the testing materials in the experiments. The potatoes were purchased in fresh. They are firm and smooth without dark spots, green areas, mold, or cuts. They are in the size of $125 \mathrm{~mm}-150 \mathrm{~mm}$ long and about $75 \mathrm{~mm}$ in diameter. The moisture content is about $4 \mathrm{~g}$ water per $5 \mathrm{~g}$ fresh potato solid. Moisture content of the sample was determined by drying thin potato slabs $(10 \mathrm{~mm}$ by $20 \mathrm{~mm}$ by $45 \mathrm{~mm}$ ) at $70^{\circ} \mathrm{C}$ under vacuum for 24 hours. The experiments were carried out in an air conditioning controlled room at temperature about $22^{\circ} \mathrm{C}$ and moisture about $70 \%$. Once a fresh potato was peeled and cut to a roughly $20 \mathrm{~mm}$ by $40 \mathrm{~mm} \times 100 \mathrm{~mm}$ brick, the experiments were performed without delay. The experimental setup is shown in Figure 12. The system consists of an ABB robot IRB 140 [27] for motion generation, speed control and distance measurement, and an ATI Force/Torque sensor ISA F/T-16 Mini40 [28] for force measurement. The robot is commanded to move at speed of $0.5 \mathrm{~mm} / \mathrm{s}$ for cutting. The force data are saved on a central computer. The forces during the cut of potatoes are shown in Figure 13.

4.1. Evaluating the Influence of Slicing Angle and Cutting Fracture Modes. When changing the slicing angle, the same shapes of the cutting force changing profiles have been obtained. The average data with standard deviation less than $0.2 \mathrm{lb}$ from 5 sets of cuts using different slicing angles on potatoes are summarized in Figure 14. According to the maximum external force when $\alpha$ is near $90^{\circ}$, where the tear mode dominates, the ultimate shear stress $\left(\tau_{u}\right)$ can be estimated as $0.18 \mathrm{lb}$. Then, using this $\tau_{u}$ and the procedure to obtain $P_{t u}$ and $P_{n u}$ in Figure 11, the theoretical fracture forces can be estimated using (12) by assuming the same $a$, $w, K_{s}, y_{u}$, and $z_{u}$ since only the slicing angle $(\alpha)$ changed 

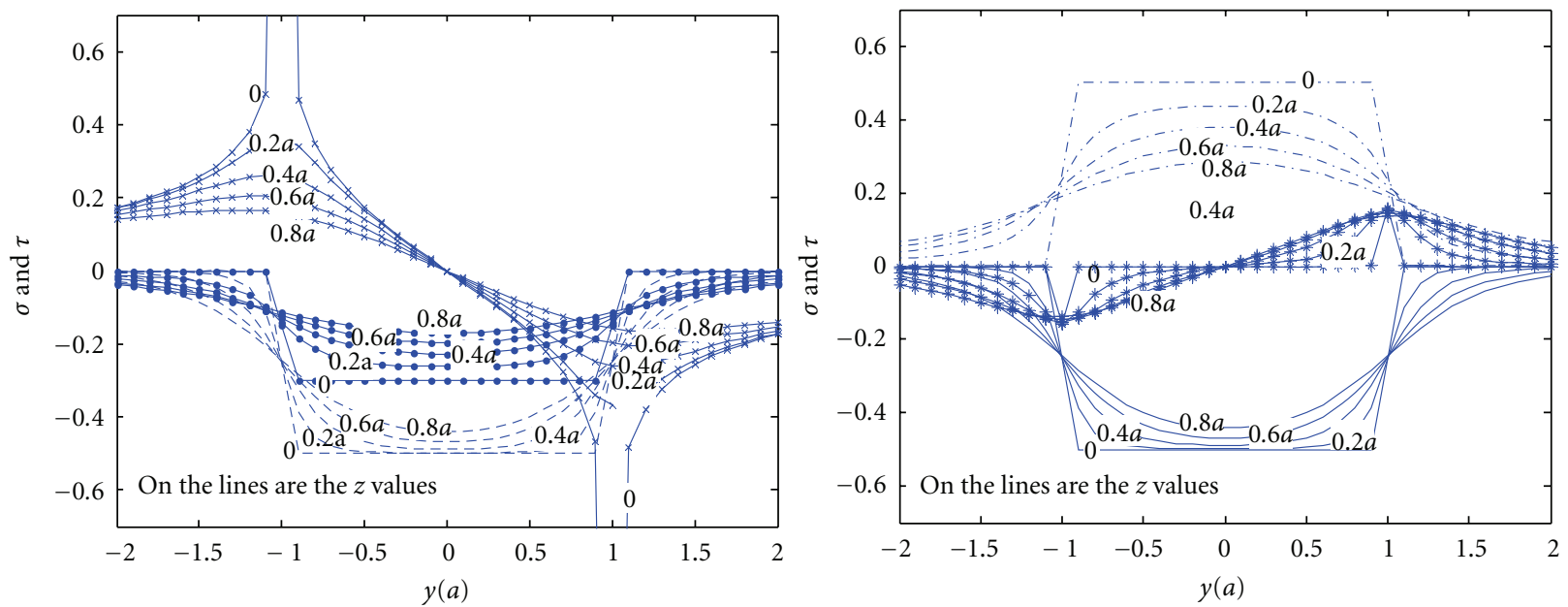

(a) $w=a$ (constant intensity)
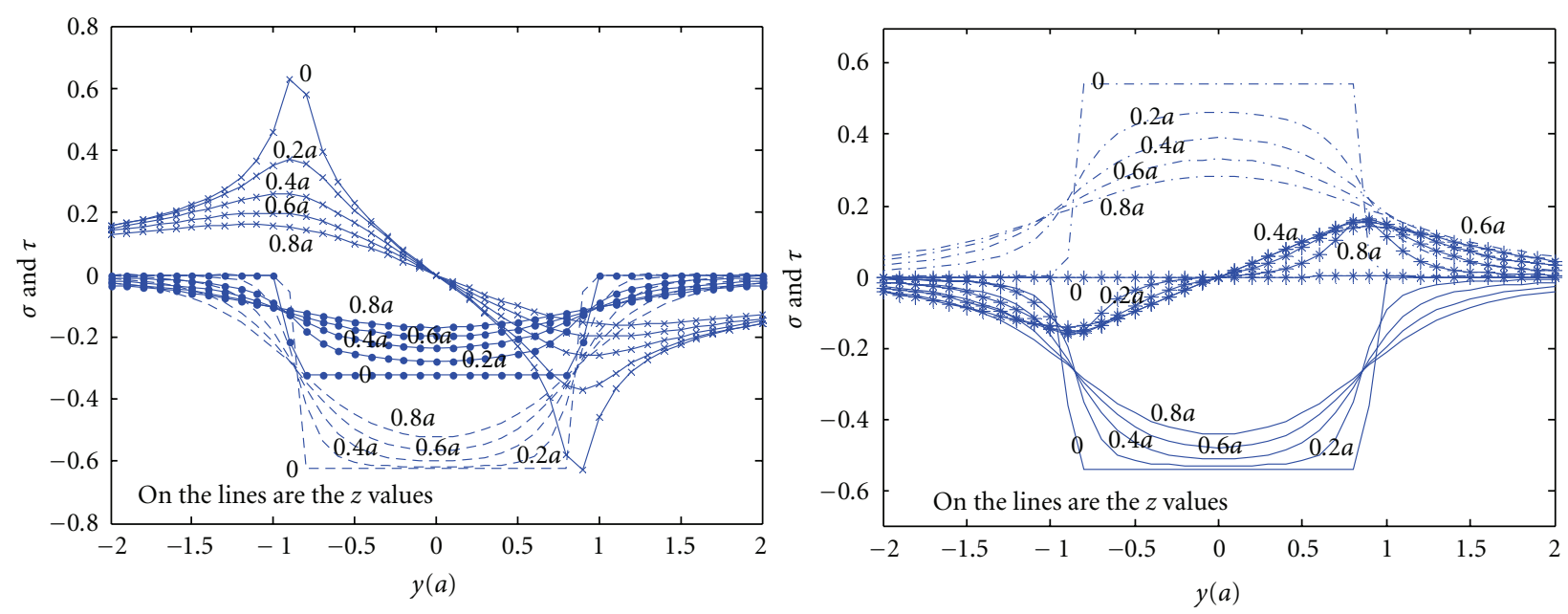

(b) $w=0.85 a$ (combined intensity)
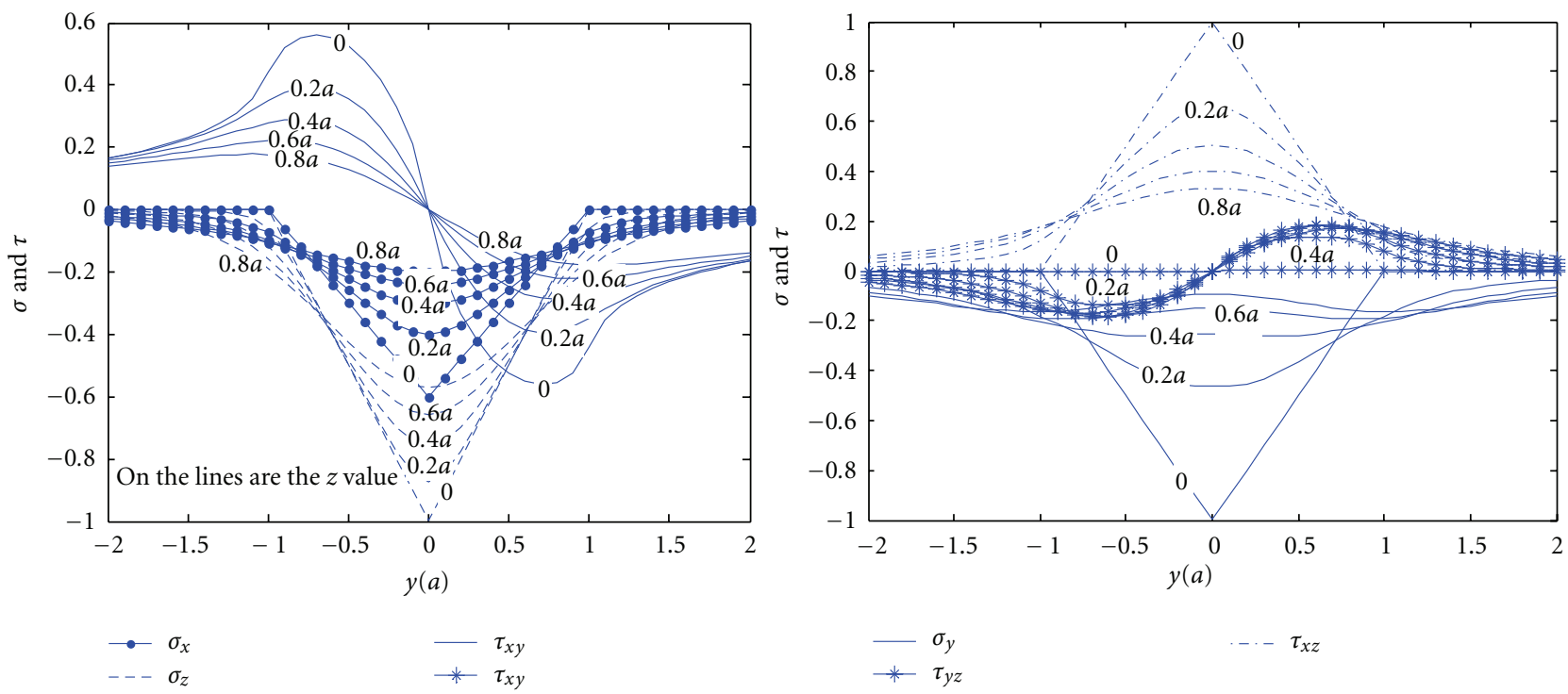

(c) $w=0$ (linear intensity)

FIgURE 8: Comparison of the normalized tensor for different cutting force profiles. 

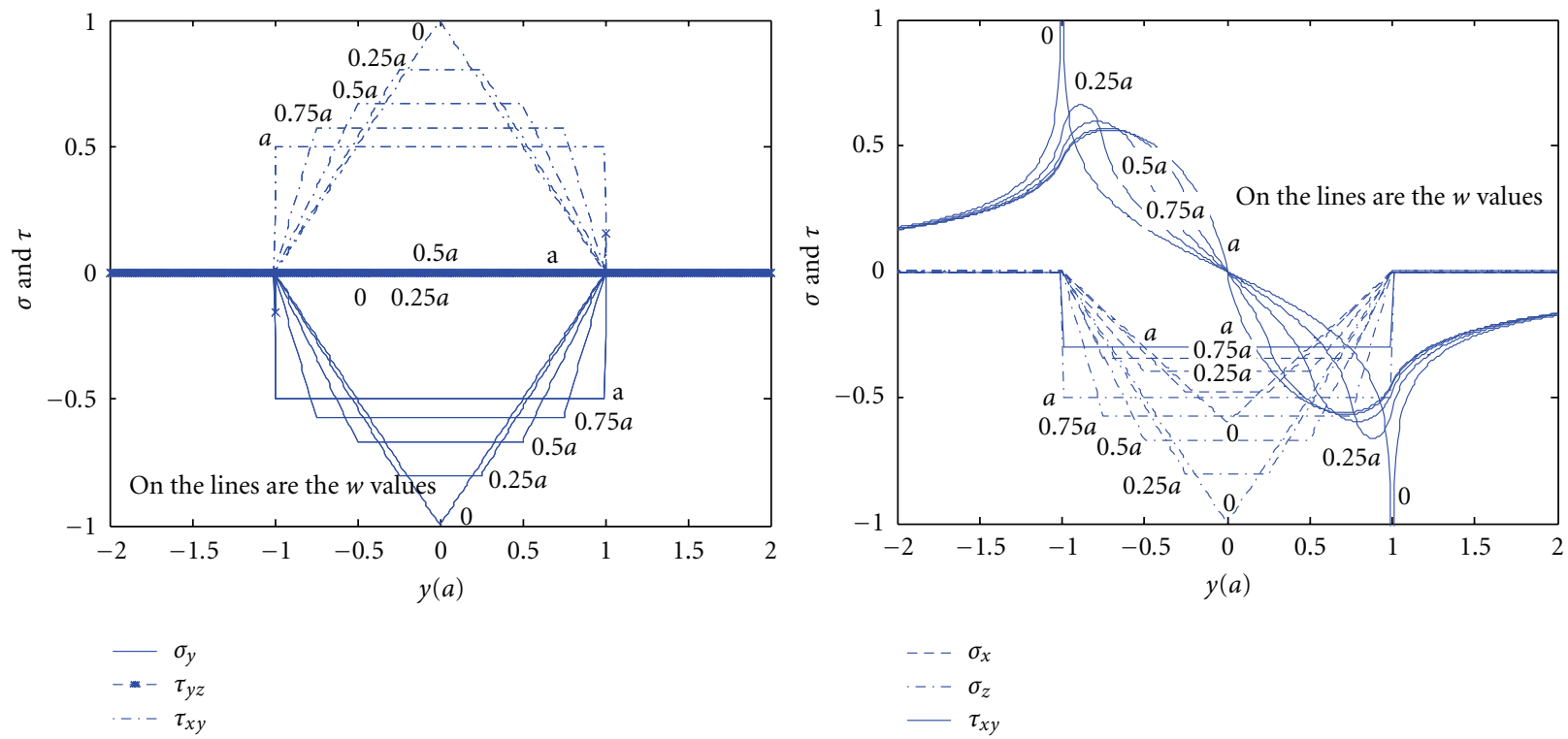

FIGURE 9: Stresses change with $w$ on the blade-material contact plane $o x y$ with same cutting force.

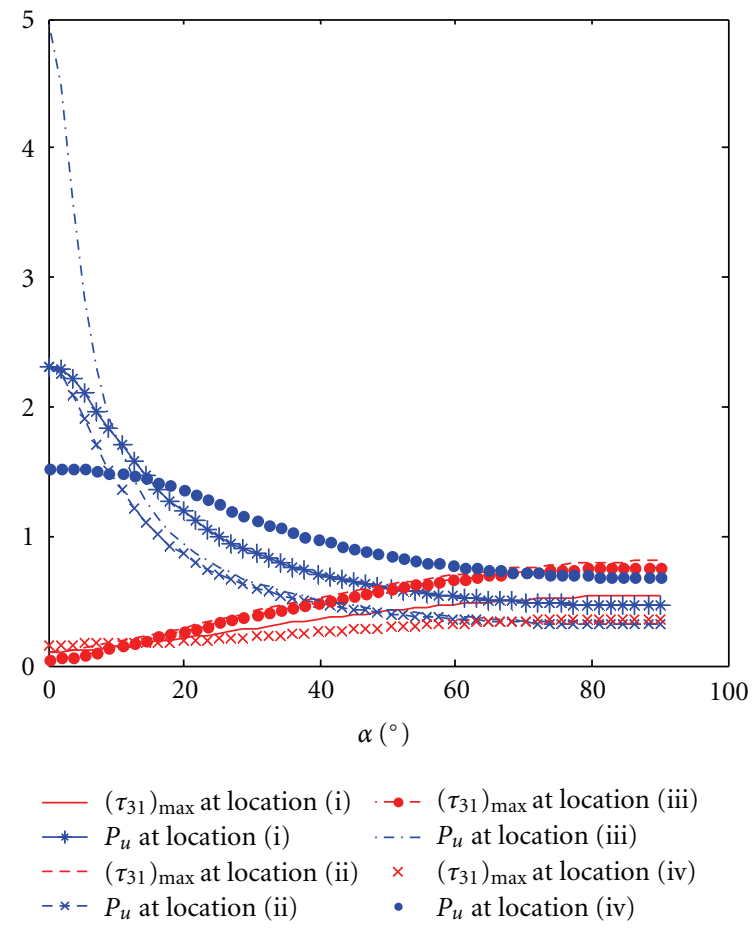

(a)

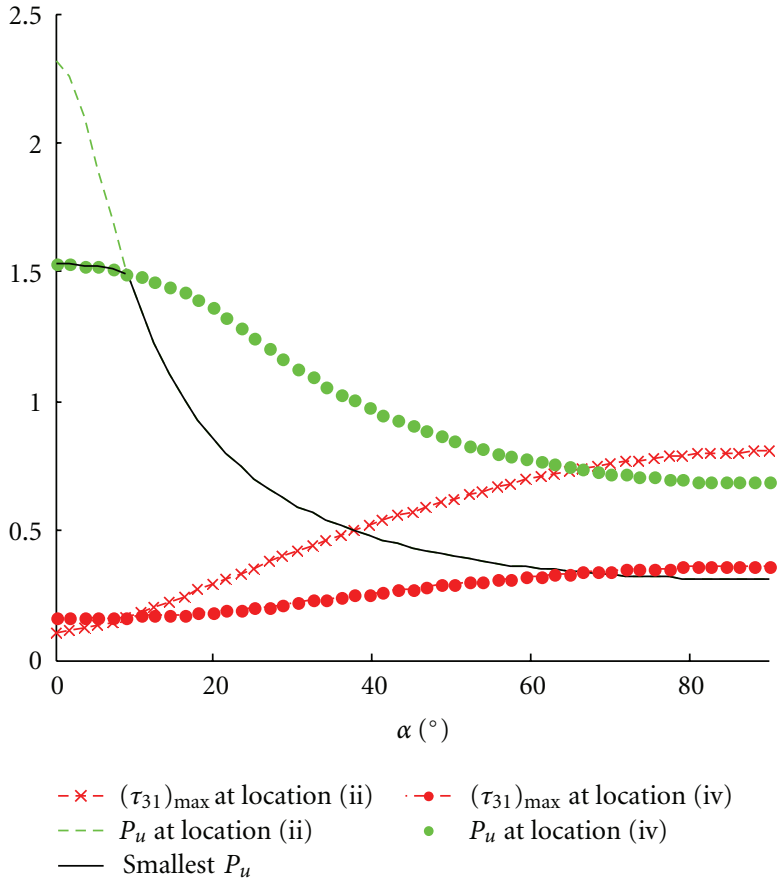

(b)

FIGURE 10: Maximum shear stresses and required minimum cutting force.

during different trails. Both of the experimental data and the theoretically estimated data are shown in Figure 14. Good match is observed.

4.2. Evaluating Relative Blade Sharpness Factor. These experiments were used to demonstrate how to implement the sharpness definition in this paper. In the experiments, blades with different sharpness were used to cut the same material to study the effect of blade sharpness. The materials (potatoes) were in rectangular shape with the same width $(13 \mathrm{~mm})$ and thickness $(30 \mathrm{~mm})$ to make sure the same contact length of each cut. The blades were prepared such that the sharpest blade and the dullest blade defined in (15)-(17) were included. 


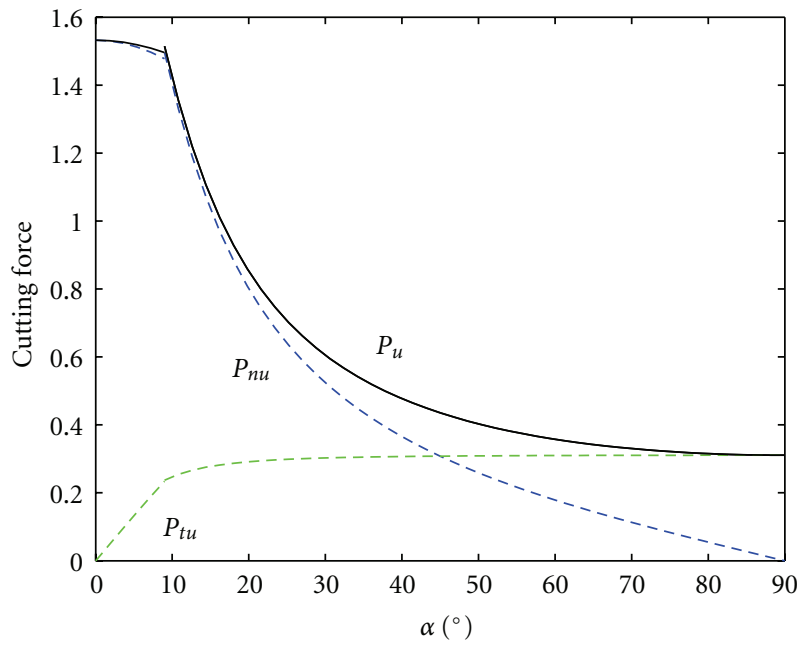

Figure 11: Total cutting force and the force components in $x$ direction $\left(P_{t u}\right)$ and $z$ direction $\left(P_{n u}\right)$.

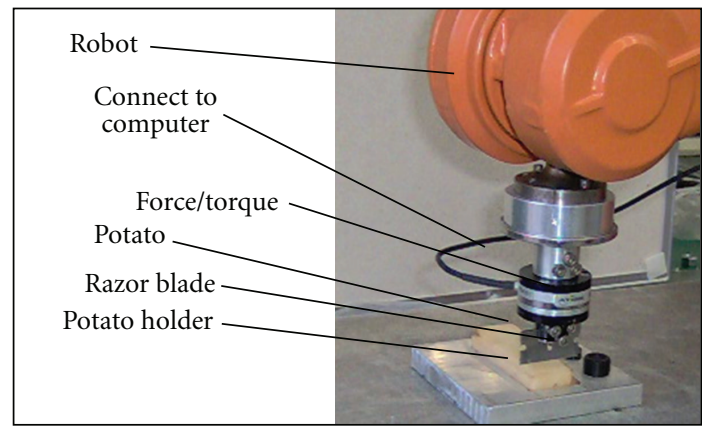

FIGURE 12: Experiment setup.

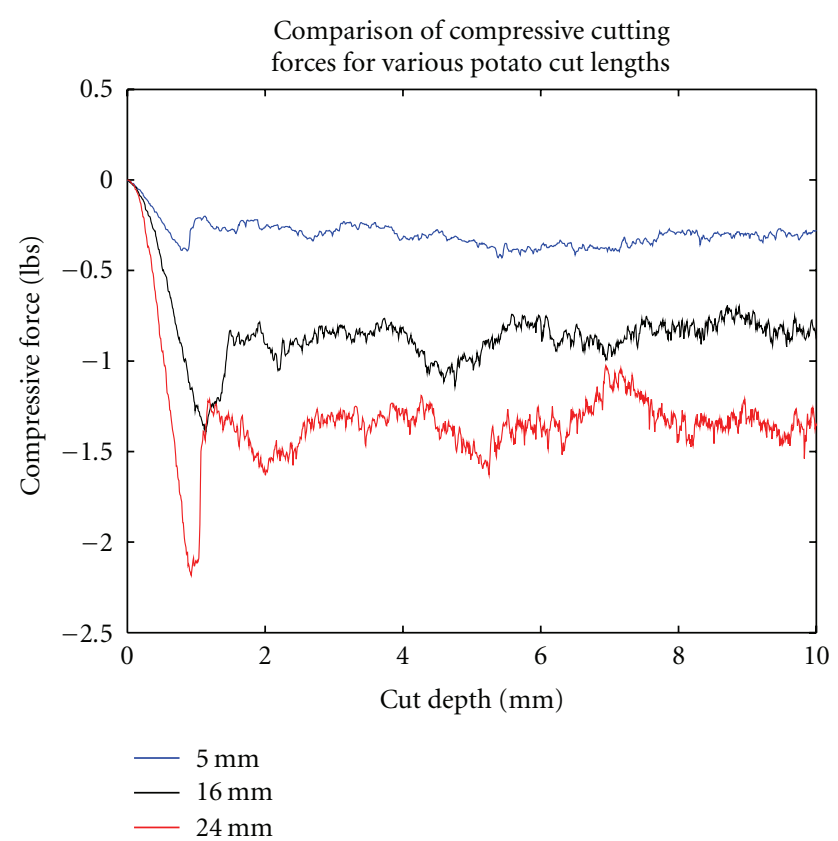

FIGURE 13: Cutting force changes with contact length.

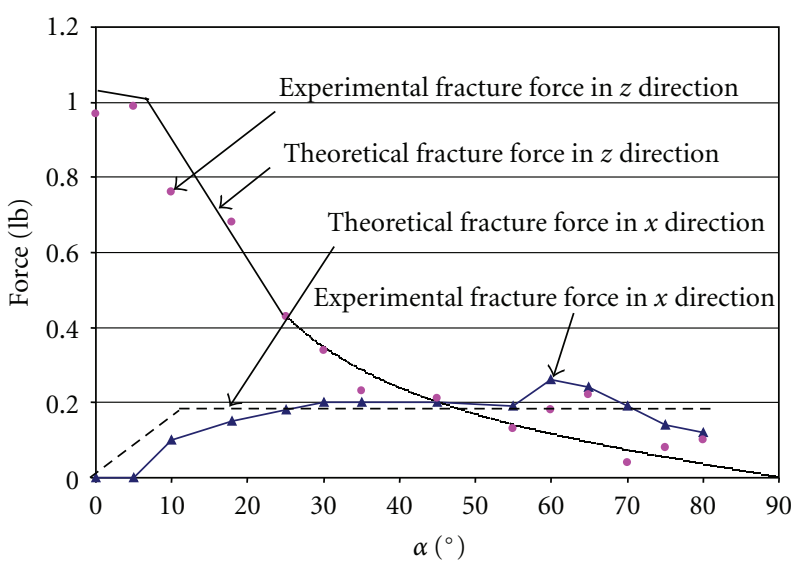

Figure 14: Experimental data and theoretical estimation of the cutting forces during potato cuts.

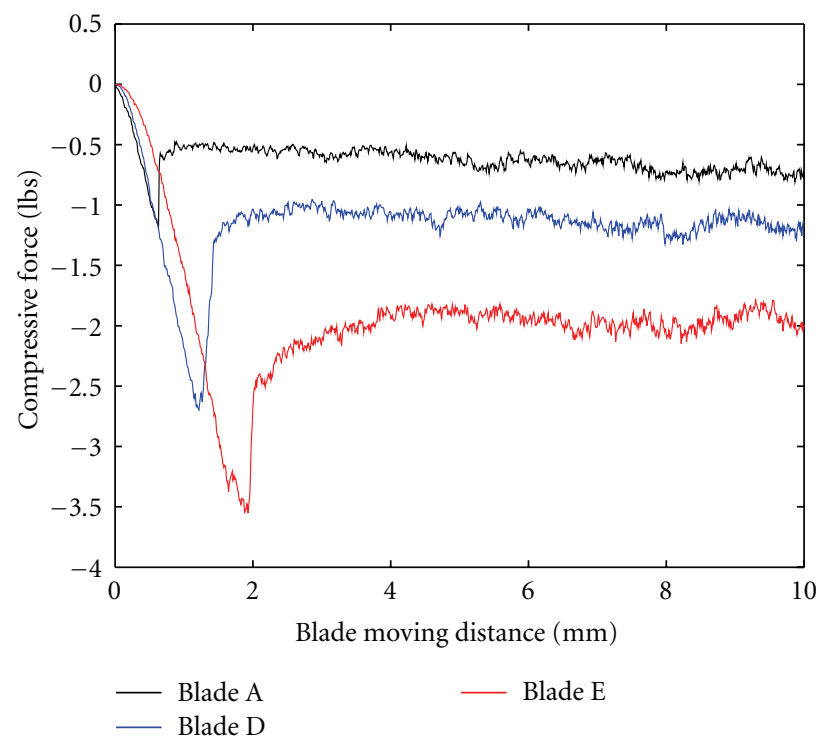

FIGURE 15: Cutting results using different sharp blades (only blades A, D, E are shown).

The procedure of the blade preparation is as follows. (1) Choose a brand-new blade (industrial-grade single-edge razor blade standard, $0.2286 \mathrm{~mm}$ thick) as the sharpest one (Blade A), define its relative sharpness $\eta_{0}=1$ and obtain its potato cutting forces; (2) use the sharpest blade (Blade A) to manually cut cardboard 30 times to form Blade B and obtain its potato cutting forces; (3) use Blade B to cut through a piece of pine wood 50 times to form Blade $\mathrm{C}$ and obtain its potato cutting forces; (4) blade $\mathrm{C}$ is dulled on aluminum by rubbing 30 times to form Blade $\mathrm{D}$ and obtain its potato cutting forces; (5) the dullest knife is obtained by dulling Blade $\mathrm{D}$ on sand paper (aluminum oxide cloth sanding sheet 80 grit) by 10 times and then obtain its potato cutting forces; (6) according to the cutting forces of the sharpest blade (Blade A) and the dullest blade (Blade E) to get $\eta_{f} ;(7)$ set sharpness level $(n)$ of Blade $\mathrm{E}$ is $5(n=5)$ and according to (18) to specify the sharpness level $\kappa$ to which 
another blade belongs. Part of the obtained force results are shown in Figure 15, and the blade sharpness level results are summarized in Table 4, where the first 4 columns show the fracture forces, Column 5 is the average fracture force, relative blade sharpness level $\eta$ and relative blade sharpness level $\kappa$ are calculated based on (13) and (18). From the last column in Table 4 , it can be seen that blades B, C, D are in the $2 \mathrm{nd}$, 3rd, and 4 th sharpness levels, respectively.

By using this method, any blade can be assigned to a certain sharpness level. When the cutting force reaches certain level by which the knife is decided to be dull, the knife needs to be resharpened or disposed.

\section{Justification}

It can be seen that the computation results from the model agree well with the potato cutting results. Noted is that the results are from the cuts of hard-crispy material. The modeling method should applicable.

Then the question is how about large deformation nonlinear materials. The mathematical model is clearly not applicable to nonlinear materials, such as meat. However, our everyday experience told us that the cuts on meat follow the rule: slicing is easier that pressing. Here, the author just provides a weak explanation of the cuts on meat: Type II fracture happens at the depth $(z)$ about 0.37 times of half of the blade width or about $z=185 \mathrm{~nm}$ if $2 a=1000 \mathrm{~nm}$. It is almost at the contact surface when you compare the whole thickness of the cutting material. Type III fracture happens at the contact surface, that is, $z=0$. In this small distance or on the contact surface, the inhomogeneity, inelasticity, and anisotropy of the cutting material will not affect the stress distribution since the react force/pressure will balance the external force statically based on Newton's third law of motion. From Figure 8, it has been checked that the stress distribution in these locations is balanced by the external force. Thus, at the moment of cutting initialization, although the model will not be valid at bigger $z$ for big deformation nonlinear materials, the model would be still valid at $z=0$ and $z \leq 0.37 a$. Since we only care about the stresses to generate the possible fracture (the results in Figure 7 are just to visualize the stress distribution yielded by the model), the stress when $z>0.37 a$ is not within our consideration and does not relate to our conclusion. Thus, the model is applicable to large deformation nonlinear materials only when $z \leq 0.37 a$. We further constrain our application range for hard-crispy materials within $z \leq 0.37 a$, that is, not all $z$.

\section{Conclusions}

In order to understand the question: why pressing-and-slicing cuts use less force than pressing-only cuts during food cutting, such as potatoes, this paper formulated and studied the change of the stress distribution with various influence factors. The cutting interaction was modeled as a belt-area force acting on the surface of a semi-infinite body. The cutting force was assumed to be in certain profile based on the observed shape of blade cutting edges. The closed form expression of the cutting interaction has been developed using the direct integration method. Compared to the knowledge in the current literature, the improvements of the modeling are twofolds: (1) this work originally expressed the cutting force (force at cutting fracture) and its change with slicing angle using mathematical equations. (2) for the first time, this work mathematically expressed the blade sharpness with blade parameters and used relative sharpness concepts based on mathematical descriptions (not based on experience) to describe blade sharpness.

The computation results were used to predict the maximum stress locations. The relationships between the applied force and slicing angle, blade edge shape, blade edge width, contact length, and the fracture force and material property were discussed. Experiments have been performed to validate the formulations. The following conclusions are drawn.

(1) The model is only applied to the moment of cutting fracture initialization. For elastic, homogeneous and isotropic material, the model is always valid. Since we are only interested in the cutting fracture, even for hard-crispy materials, the applicable range in material is only $z \leq 0.37 a$.

(2) During the cut with slicing angle smaller than $10^{\circ}$, or pressing-only or mainly pressing cuts, blade cutting is a type II fracture due to the shear stress $\tau_{y z}$. With slicing angle bigger than $10^{\circ}$, or called pressing-andslicing cuts, blade cutting is a type III fracture due to the shear stress $\tau_{x y}$ and $\tau_{x z}$. Type III fracture uses considerable less force than type II fracture. This answered why pressing-and-slicing cuts use less force than pressing-only cuts. However, cuts with bigger slicing angle will make the cutting feeding less. In order to keep work efficiency, an optimal slicing angle should be selected to satisfy both minimum cutting force and maximum cut feeding speed.

(3) The shape of blade cutting edge determines the distribution of the cutting force on the contact surface between the blade and the cutting material. The cutting force distribution profile determines the sharpness of a blade. By using the relative sharpness factor concept, blade sharpness can be quantified based on the obtained forces from sharpest blades and dullest blades.

(4) It can be observed from both the computation and experimental results that the cutting force $\left(P_{u}\right)$ is proportional to the contact length $(l)$. This obvious observation agrees with those from everyday life.

(5) Edge shape and edge width have the combined influence on fracture force. For the same edge width, external force is proportional to the maximum force intensity, which the edge shape can generate.

(6) Based on material properties, the knife sharpness properties and the interaction between the blade and the material, the required force to realize certain cuts can be predicted. This observation provides the principle to optimize the cutting mechanism design and 
TABLE 4: Blade relative sharpness level.

\begin{tabular}{|c|c|c|c|c|c|c|c|}
\hline Blade & & Force at & $\operatorname{ture}(1$ & & Average force $(N)$ & $\eta$ (relative sharpness factor) & $\kappa$ (relative sharpness level) \\
\hline A & 5.20 & 6.49 & 6.36 & 6.67 & 6.18 & 1 & 1 \\
\hline B & 7.87 & 7.16 & 7.92 & 7.78 & 7.70 & 1.24 & 2 \\
\hline $\mathrm{C}$ & 10.23 & 9.65 & 10.19 & 10.76 & 10.19 & 1.65 & 3 \\
\hline $\mathrm{D}$ & 12.01 & 11.17 & 12.14 & 11.57 & 11.74 & 1.89 & 4 \\
\hline $\mathrm{E}$ & 15.79 & 15.75 & 16.28 & 15.35 & 15.79 & 2.55 & 5 \\
\hline
\end{tabular}

the force control algorithm design for the automation of the cutting operations.

Regarding the future work, the stress intensity factors $K_{\mathrm{II}}$ and $K_{\text {III }}$ will be analyzed and quantified. The influence of the relative moving speed will also be investigated. On the long term, the research work will try to understand and model the behavior of interesting biomaterials or hybrid materials during robot-controlled cuts. Then robots can be intelligent to adapt themselves for any deformation of material and any varieties of material's structure.

\section{Appendix}

\section{A. Derivation of Stress Distribution dut to Various Forces}

\section{A.1. Stress Distribution due to Point Force}

(1) Normal Point Force Only. When there is only normal point force $P_{p t}$, for material with Poisson's ratio $\mu$, the normal stress $\sigma$ and the shear stress $\tau$ at point $A(x, y, z)$ are given as

$$
\left[\sigma^{n p}\right]=\left[\begin{array}{cccccc}
\sigma_{x}^{n p} & \sigma_{y}^{n p} & \sigma_{z}^{n p} & \tau_{x y}^{n p} & \tau_{y z}^{n p} & \tau_{x z}^{n p}
\end{array}\right]^{T},
$$

where

$$
\begin{aligned}
& \sigma_{x}^{n p}= {\left[\frac{(1-2 \mu) R}{R+z}-3 \frac{r^{2} z}{R^{3}}\right] \frac{x^{2}}{r^{2}} } \\
&+(1-2 \mu)\left[\frac{z}{R}-\frac{R}{R+z}\right] \frac{y^{2}}{r^{2}} \frac{P_{p n}}{2 \pi R^{2}}, \\
& \sigma_{y}^{n p}= {\left[\frac{(1-2 \mu) R}{R+z}-3 \frac{r^{2} z}{R^{3}}\right] \frac{y^{2}}{r^{2}} } \\
&+(1-2 \mu)\left[\frac{z}{R}-\frac{R}{R+z}\right] \frac{x^{2}}{r^{2}} \frac{P_{p n}}{2 \pi R^{2}}, \\
& \tau_{x y}^{n p}=\left\{\left[\frac{(1-2 \mu) R}{R+z}-3 \frac{r^{2} z}{R^{3}}\right]\right. \\
&\left.-(1-2 \mu)\left[\frac{z}{R}-\frac{R}{R+z}\right]\right\} \frac{x y}{r^{2}} \frac{P_{p n}}{2 \pi R^{2}}, \\
& \sigma_{z}^{n p}=\frac{3 z^{3}}{R^{3}} \frac{P_{p n}}{2 \pi R^{2}}, \\
& \tau_{y z}^{n p}=-\frac{3 y z^{2}}{R^{3}} \frac{P_{p n}}{2 \pi R^{2}}, \\
& \tau_{x z}^{n p}=-\frac{3 x z^{2}}{R^{3}} \frac{P_{p n}}{2 \pi R^{2}} .
\end{aligned}
$$

The superscript $n p$ (stands for normal direction and on a point) in (A.1a) to (A.1g) represents the stresses generated by external force $P_{p n}$.

(2) Tangential Point Force Only. When there is only tangential point force $P_{p t}$, the stress distribution is known as the Cerruti solution and is given in [8] as

$$
\left[\sigma^{t p}\right]=\left[\begin{array}{llllll}
\sigma_{x}^{t p} & \sigma_{y}^{t p} & \sigma_{z}^{t p} & \tau_{z x y}^{t p} & \tau_{y z}^{t p} & \tau_{x z}^{t p}
\end{array}\right]
$$

where

$$
\begin{gathered}
\sigma_{x}^{t p}=x\left[\frac{(1-2 \mu)}{(R+z)^{2}}\left(R^{2}-y^{2}-\frac{2 R y^{2}}{R+z}\right)-\frac{3 x^{2}}{R^{2}}\right] \frac{P_{p t}}{2 \pi R^{3}}, \\
\sigma_{y}^{t p}=x\left[\frac{(1-2 \mu)}{(R+z)^{2}}\left(3 R^{2}-x^{2}-\frac{2 R x^{2}}{R+z}\right)-\frac{3 y^{2}}{R^{2}}\right] \frac{P_{p t}}{2 \pi R^{3}}, \\
\tau_{x z}^{t p}=y\left[\frac{(1-2 \mu)}{(R+z)^{2}}\left(-R^{2}+x^{2}+\frac{2 R x^{2}}{R+z}\right)-\frac{3 x^{2}}{R^{2}}\right] \frac{P_{p t}}{2 \pi R^{3}}, \\
\sigma_{z}^{t p}=-\frac{3 x z^{2}}{R^{2}} \frac{P_{p t}}{2 \pi R^{3}}, \\
\tau_{x y}^{t p}=-\frac{3 x y z}{R^{2}} \frac{P_{p t}}{2 \pi R^{3}}, \\
\tau_{y z}^{t p}=-\frac{3 x^{2} z}{R^{2}} \frac{P_{p t}}{2 \pi R^{3}} .
\end{gathered}
$$

The superscript $t p$ in (A.2a) to (A.2g) represents the stresses generated by external force $P_{p t}$.

\section{A.2. Stress Distribution due to Line Force}

(1) Normal Line Force Only. The closed form solution of the stresses at any point $(x, y, z)$ in the semi-infinite body generated by line force acting along $\left(x_{1}, x_{2}\right)$ are shown in (A.3). 


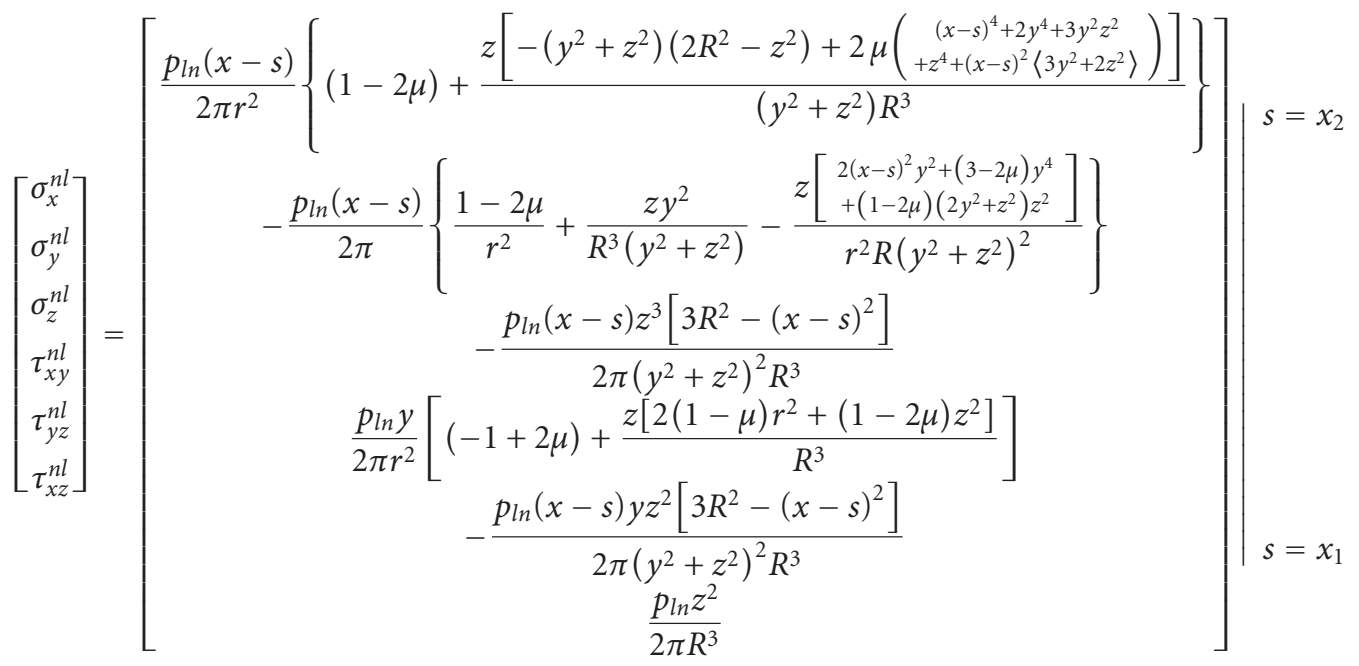

When $x_{1} \rightarrow-\infty$ and $x_{2} \rightarrow \infty$, the stress tensor is

$$
\begin{aligned}
& {\left[\begin{array}{llllll}
\sigma_{x}^{n l \infty} & \sigma_{y}^{n l \infty} & \sigma_{z}^{n l \infty} & \tau_{x y}^{n l \infty} & \tau_{y z}^{n l \infty} & \tau_{x z}^{n l \infty}
\end{array}\right]} \\
& \quad=\frac{2 p_{\ln } z}{\pi\left(y^{2}+z^{2}\right)}\left[\begin{array}{lllll}
-\mu-\frac{y^{2}}{y^{2}+z^{2}} & -\frac{z^{2}}{y^{2}+z^{2}} & 0 & \frac{y z}{y^{2}+z^{2}} & 0
\end{array}\right] .
\end{aligned}
$$

(2) Tangential Line Force Only. The closed-form expression of the stress distribution by a tangential force on line segment $\left[x_{1}, x_{2}\right]$ is given in (A.5).

$$
\left[\begin{array}{c}
\sigma_{x}^{t l} \\
\sigma_{y}^{t l} \\
\sigma_{z}^{t l} \\
\tau_{x y}^{t l} \\
\tau_{y z}^{t l} \\
\tau_{x z}^{t l}
\end{array}\right]=\left[\begin{array}{c}
\frac{p_{l t}}{2 \pi}\left(-\frac{y^{2}+z^{2}}{R^{3}}+\frac{(1-2 \mu) y^{2}+3 z^{2}}{z^{2} R}-\frac{(1-2 \mu) y^{2}}{z(z+R)^{2}}-\frac{(1-2 \mu)\left(y^{2}+z^{2}\right)}{z^{2}(z+R)}\right) \\
f\left(\sigma_{y}\right) \\
\frac{p_{l t} z^{2}}{2 \pi R^{3}} \\
f\left(\tau_{x y}\right) \\
\frac{p_{l t} y z}{2 \pi R^{3}} \\
-\frac{p_{l t} z}{2 \pi\left(y^{2}+z^{2}\right)} \frac{(x-s)^{3}}{R^{3}}
\end{array}\right] \mid s=x_{2}
$$

where

$$
\begin{aligned}
& f\left(\sigma_{y}\right)=\frac{p l t}{2 \pi r^{4}}\left(\frac{1}{R^{3}}\left\{\begin{array}{c}
2(1-2 \mu) y^{2} z-(1-2 \mu)\left((x-s)^{2}+y^{2}\right) z+ \\
{\left[z^{2}+2 \mu\left(y^{2}-z^{2}\right)\right] s^{4}+\left[8 u x\left(-y^{2}+z^{2}\right)-4 z^{2} x\right] s^{3}+} \\
\left\{2 u\left[2 y^{4}+y^{2} z^{2}-z^{4}+6 x^{2}\left(y^{2}-z^{2}\right)\right]+z^{2}\left(6 x^{2}-y^{2}+z^{2}\right)\right\} s^{2} \\
\left.+4 \mu x\left[2 y^{4}+y^{2} z^{2}-z^{4}+2 x^{2}\left(y^{2}-z^{2}\right)\right]-2 z^{2} x^{2}\left(2 x^{2}-y^{2}+z^{2}\right)\right\} s \\
+z^{2}\left[x^{4}+x^{2}\left(-y^{2}+z^{2}\right)-y^{2}\left(2 y^{2}+z^{2}\right)\right] \\
+2 \mu\left[x^{2}\left(2 y^{4}+y^{2} z^{2}-z^{4}\right)+x^{4}\left(y^{2}-z^{2}\right)+y^{2}\left(y^{2}+z^{2}\right)^{2}\right]
\end{array}\right\}\right)
\end{aligned}
$$

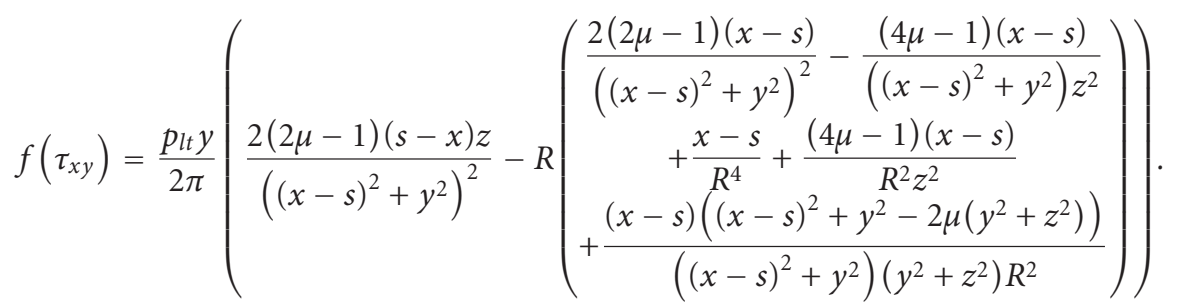


As $x_{1}$ and $x_{2}$ go to $-\infty$ and $\infty$, respectively, that is, a line force acting on the boundary of a semi-infinite body, the stress distribution changes to

$$
\begin{aligned}
& {\left[\begin{array}{llllll}
\sigma_{x}^{t l \infty} & \sigma_{y}^{t l \infty} & \sigma_{z}^{t l \infty} & \tau_{x y}^{t l \infty} & \tau_{y z}^{t l \infty} & \tau_{x z}^{t l \infty}
\end{array}\right]} \\
& \quad=\frac{p_{l t}}{\pi\left(y^{2}+z^{2}\right)}\left[\begin{array}{llllll}
0 & 0 & 0 & -y & 0 & z
\end{array}\right] .
\end{aligned}
$$

\section{A.3. Stress Distribution due to Area Force.}

(1) Normal Area Force Only. The stresses generated by the normal force intensity between $[-a,-w]$ are

$$
\begin{aligned}
& f_{11 n}=\sigma_{x}^{n 1}=\left\{-\frac{2 \mu}{\pi} \frac{q_{n}}{a-w}\left((y+a) T+\frac{z}{2} \Gamma\right)\right\} \mid \begin{array}{l}
v=-w, \\
v=-a,
\end{array} \\
& f_{21 n}=\sigma_{y}^{n 1}=\left\{\frac { - 1 } { \pi } \frac { q _ { n } } { a - w } \left(\frac{z\left[a(y-t) v+\left(-t y+y^{2}+z^{2}\right)\right]}{(y-v)^{2}+z^{2}}\right.\right. \\
& \left.\left.+(a+y) T+\frac{z}{2} \Gamma\right)\right\} \mid \begin{array}{l}
v=-w, \\
v=-a,
\end{array} \\
& f_{31 n}=\sigma_{z}^{n 1}=\left\{\frac { - 1 } { \pi } \frac { q _ { n } } { a - w } \left(\frac{z\left[a(v-y)+\left(-v y+y^{2}+z^{2}\right)\right]}{(y-v)^{2}+z^{2}}\right.\right. \\
& +(a+y) T)\}\left\{\begin{array}{l}
v=-w \\
v=-a
\end{array}\right. \\
& f_{4 n}=\tau_{x y}^{n 1}=0 \\
& f_{51 n}=\tau_{y z}^{n 1}=\left\{\frac{z}{\pi} \frac{q_{v}}{a-w}\left[\frac{z(a+v)}{(v-y)^{2}+z^{2}}-T\right]\right\} \mid \begin{array}{l}
v=-w \\
v=-a
\end{array}, \\
& f_{61 n}=\tau_{x z}^{n 1}=0,
\end{aligned}
$$

where $T=\tan ^{-1}[(y-v) / z], \Gamma=\ln \left((v-y)^{2}+z^{2}\right)$ and $\{f(v)\} \mid \begin{gathered}v=-w \\ v=-a\end{gathered}=f(-w)-f(-a)$.

The stresses generated by the normal force intensity between $(-w, w)$ are obtained as

$$
\begin{aligned}
& f_{12 n}=\sigma_{x}^{n 2}=\left\{\frac{2 q_{n} \mu}{\pi} T\right\} \mid \begin{array}{l}
v=w, \\
v=-w,
\end{array} \\
& f_{22 n}=\sigma_{y}^{n 2}=\left\{\frac{-q_{n}}{\pi}\left(\frac{(y-v) z}{(y-v)^{2}+z^{2}}+T\right)\right\} \mid \begin{array}{l}
v=w, \\
v=-w,
\end{array} \\
& f_{32 n}=\sigma_{z}^{n 2}=\left\{\frac{-q_{n}}{\pi}\left(\frac{(v-y) z}{(v-y)^{2}+z^{2}}+T\right)\right\} \mid \begin{array}{l}
v=w, \\
v=-w,
\end{array} \\
& f_{42 n}=\tau_{x y}^{n 2}=0, \\
& f_{52 n}=\tau_{y z}^{n 2}=\left\{\frac{q_{n} z^{2}}{\pi(v-y)^{2}+z^{2}}\right\} \mid \begin{array}{l}
v=w, \\
v=-w,
\end{array} \\
& f_{62 n}=\tau_{x z}^{n 2}=0 .
\end{aligned}
$$

The stresses generated by the normal force intensity between $[w, a]$ are obtained as

$$
\begin{aligned}
& f_{13 n}=\sigma_{x}^{n 3}=\left\{-\frac{2 \mu}{\pi} \frac{q_{n}}{w-a}\left((y-a) T+\frac{z}{2} \Gamma\right)\right\} \mid \begin{array}{l}
v=a, \\
v=w,
\end{array} \\
& f_{23 n}=\sigma_{y}^{n 3}=\left\{\frac { - 1 } { \pi } \frac { q _ { n } } { w - a } \left(\frac{z\left[a(y-v)+\left(-v y+y^{2}+z^{2}\right)\right]}{(y-v)^{2}+z^{2}}\right.\right. \\
& \left.\left.+(y-a) T+\frac{z}{2} \Gamma\right)\right\} \mid \begin{array}{l}
v=a, \\
v=w,
\end{array}
\end{aligned}
$$

The stress tensor generated by normal force can then be expressed as

$$
\begin{aligned}
{\left[\begin{array}{lll}
\sigma_{x}^{n} & \tau_{x y}^{n} & \tau_{x z}^{n} \\
\tau_{x y}^{n} & \sigma_{y}^{n} & \tau_{y z}^{n} \\
\tau_{x z}^{n} & \tau_{y z}^{n} & \sigma_{z}^{n}
\end{array}\right] } & =\left[\begin{array}{lll}
\sum_{i=1}^{3} \sigma_{x}^{n i} & \sum_{i=1}^{3} \tau_{x y}^{n i} & \sum_{i=1}^{3} \tau_{x z}^{n i} \\
\sum_{i=1}^{3} \tau_{x y}^{n i} & \sum_{i=1}^{3} \sigma_{y}^{n i} & \sum_{i=1}^{3} \tau_{y z}^{n i} \\
\sum_{i=1}^{3} \tau_{x z}^{n i} & \sum_{i=1}^{3} \tau_{y z}^{n i} & \sum_{i=1}^{3} \sigma_{y}^{n i}
\end{array}\right] \\
& =\left[\begin{array}{lll}
\sum_{i=1}^{3} f_{1 i n} & \sum_{i=1}^{3} f_{4 i n} & \sum_{i=1}^{3} f_{6 i n} \\
\sum_{i=1}^{3} f_{4 i n} & \sum_{i=1}^{3} f_{2 i n} & \sum_{i=1}^{3} f_{5 i n} \\
\sum_{i=1}^{3} f_{6 i n} & \sum_{i=1}^{3} f_{5 i n} & \sum_{i=1}^{3} f_{3 i n}
\end{array}\right] .
\end{aligned}
$$

(2) Tangential Area Force Only. The stresses generated by the tangential force intensity between $[-a,-w]$ are obtained as

$$
\begin{aligned}
& f_{11 t}=\sigma_{x}^{t}=0, \\
& f_{21 t}=\sigma_{y}^{t}=0, \\
& f_{31 t}=\sigma_{z}^{t}=0, \\
& f_{41 t}=\tau_{x y}^{t}=\left\{\frac{1}{\pi} \frac{q t}{a-w} v\left(t-z T+\frac{(a+y)}{2} \Gamma\right)\right\} \mid \begin{array}{l}
v=-w, \\
v=-a,
\end{array}
\end{aligned}
$$




$$
\begin{aligned}
& f_{51 t}=\tau_{y z}^{t}=0 \\
& f_{61 t}=\tau_{x z}^{t}=\left\{\frac{1}{\pi} \frac{q_{t}}{a-w}\left((a+y) T+\frac{z}{2} \Gamma\right)\right\} \mid \begin{array}{l}
v=-w \\
v=-a
\end{array}
\end{aligned}
$$

The stresses generated by the tangential force intensity between $(-w, w)$ are obtained as

$$
\begin{aligned}
& f_{12 t}=\sigma_{x}^{t}=0, \\
& f_{22 t}=\sigma_{y}^{t}=0, \\
& f_{32 t}=\sigma_{z}^{t}=0, \\
& f_{42 t}=\tau_{x y}^{t}=\left\{\frac{q_{t}}{2 \pi} \Gamma\right\} \mid \begin{array}{l}
v=w, \\
v=-w,
\end{array} \\
& f_{52 t}=\tau_{y z}^{t}=0, \\
& f_{62 t}=\tau_{x z}^{t}=\left\{\frac{q_{t}}{\pi} T\right\} \mid \begin{array}{l}
v=w . \\
v=-w .
\end{array}
\end{aligned}
$$

The stresses generated by the tangential force intensity between $[w, a]$ are obtained as

$$
\begin{aligned}
& f_{13 t}=\sigma_{x}^{t}=0, \\
& f_{23 t}=\sigma_{y}^{t}=0 \\
& f_{33 t}=\sigma_{z}^{t}=0 \\
& f_{43 t}=\tau_{x y}^{t}=\left\{\frac{1}{\pi} \frac{q_{t}}{w-a}\left(v-z T+\frac{(-a+y)}{2} \Gamma\right)\right\} \mid \begin{array}{l}
v=a, \\
v=w,
\end{array} \\
& f_{53 t}=\tau_{y z}^{t}=0, \\
& f_{63 t}=\tau_{x z}^{t}=\left\{\frac{1}{\pi} \frac{q_{t}}{w-a}\left((-a+y) T+\frac{z}{2} \Gamma\right)\right\} \mid \begin{array}{l}
v=a . \\
v=w .
\end{array}
\end{aligned}
$$

The final stress tensor generated by tangential force can then be expressed as

$$
\begin{aligned}
{\left[\begin{array}{ccc}
\sigma_{x}^{t} & \tau_{x y}^{t} & \tau_{x z}^{t} \\
\tau_{x y}^{t} & \sigma_{y}^{t} & \tau_{y z}^{t} \\
\tau_{x z}^{t} & \tau_{y z}^{t} & \sigma_{z}^{t}
\end{array}\right] } & =\left[\begin{array}{lll}
\sum_{i=1}^{3} \sigma_{x}^{t i} & \sum_{i=1}^{3} \tau_{x y}^{t i} & \sum_{i=1}^{3} \tau_{x z}^{t i} \\
\sum_{i=1}^{3} \tau_{x y}^{t i} & \sum_{i=1}^{3} \sigma_{y}^{t i} & \sum_{i=1}^{3} \tau_{y z}^{t i} \\
\sum_{i=1}^{3} \tau_{x z}^{t i} & \sum_{i=1}^{3} \tau_{y z}^{t i} & \sum_{i=1}^{3} \sigma_{y}^{t i}
\end{array}\right] \\
& =\left[\begin{array}{ccc}
3 & 3 \\
\sum_{i=1}^{3} f_{1 i t} & \sum_{i=1}^{3} f_{4 i t} & \sum_{i=1}^{3} f_{6 i t} \\
\sum_{i=1}^{3} f_{4 i t} & \sum_{i=1}^{3} f_{2 i t} & \sum_{i=1}^{3} f_{5 i t} \\
\sum_{i=1}^{3} f_{6 i t} & \sum_{i=1}^{3} f_{5 i t} & \sum_{i=1}^{3} f_{3 i t}
\end{array}\right] .
\end{aligned}
$$

(3) Force with Both Normal and Tangential Components. Using (A.11) and (A.15), the stress distribution generated by both $q_{n}$ and $q_{t}$ is obtained as

$$
\begin{aligned}
& {\left[\begin{array}{ccc}
\sigma_{x} & \tau_{x y} & \tau_{x z} \\
\tau_{x y} & \sigma_{y} & \tau_{y z} \\
\tau_{x z} & \tau_{y z} & \sigma_{z}
\end{array}\right] } \\
&=\left[\begin{array}{lll}
\sigma_{x}^{n} & \tau_{x y}^{n} & \tau_{x z}^{n} \\
\tau_{x y}^{n} & \sigma_{y}^{n} & \tau_{y z}^{n} \\
\tau_{x z}^{n} & \tau_{y z}^{n} & \sigma_{z}^{n}
\end{array}\right]+\left[\begin{array}{ccc}
\sigma_{x}^{t} & \tau_{x y}^{t} & \tau_{x z}^{t} \\
\tau_{x y}^{t} & \sigma_{y}^{t} & \tau_{y z}^{t} \\
\tau_{x z}^{t} & \tau_{y z}^{t} & \sigma_{z}^{t}
\end{array}\right] \\
&= {\left[\begin{array}{llll}
\sum_{i=1}^{3}\left(f_{1 i n}+f_{1 i t}\right) & \sum_{i=1}^{3}\left(f_{4 i n}+f_{4 i t}\right) & \sum_{i=1}^{3}\left(f_{6 i n}+f_{6 i t}\right) \\
\sum_{i=1}^{3}\left(f_{4 i n}+f_{4 i t}\right) & \sum_{i=1}^{3}\left(f_{2 i n}+f_{2 i t}\right) & \sum_{i=1}^{3}\left(f_{5 i n}+f_{5 i t}\right) \\
\sum_{i=1}^{3}\left(f_{6 i n}+f_{6 i t}\right) & \sum_{i=1}^{3}\left(f_{5 i n}+f_{5 i t}\right) & \sum_{i=1}^{3}\left(f_{3 i n}+f_{3 i t}\right)
\end{array}\right] . }
\end{aligned}
$$

Equation (A.16) can be rewritten as

$$
\begin{gathered}
{\left[\begin{array}{lll}
\sigma_{x} & \tau_{x y} & \tau_{x z} \\
\tau_{x y} & \sigma_{y} & \tau_{y z} \\
\tau_{x z} & \tau_{y z} & \sigma_{z}
\end{array}\right]} \\
\quad=\left[\begin{array}{lll}
q_{n} f_{1}(y, z, a, w) & q_{t} f_{4}(y, z, a, w) & q_{t} f_{5}(y, z, a, w) \\
q_{t} f_{4}(y, z, a, w) & q_{n} f_{2}(y, z, a, w) & q_{n} f_{6}(y, z, a, w) \\
q_{t} f_{5}(y, z, a, w) & q_{n} f_{6}(y, z, a, w) & q_{n} f_{3}(y, z, a, w)
\end{array}\right],
\end{gathered}
$$

where the functions $f_{1}, f_{2}, f_{3}, f_{4}, f_{5}$, and $f_{6}$ can be explicitly expressed using (A.8) to (A.10) and (A.12) to (A.14) using the variables $y, z, a, w$.

\section{Symbols}

a: $\quad$ Half of the width of the blade edge (see Figure 3)

$A(x, y, z):$ A point in the material with coordinate $(x, y, z)$ in frame $o x y z$ (see Figure 3 )

$K_{s}: \quad$ Ratio of the fracture initialization force over continuous cutting force

$l$ : $\quad$ Contact length between the blade and the material (see Figure 3)

$p_{n}$ and $p_{t}$ : Cutting pressure components in the $z$ (normal) direction and $x$ (tangential) direction, respectively (see Table 2 )

$p_{l n}$ and $p_{l t}$ : Normal and tangential components of line distributed cutting force 


\begin{tabular}{|c|c|}
\hline$P:$ & tting force (see Figure 4 and Table 2) \\
\hline$P_{c}:$ & rce during continuous cutting fracture \\
\hline$P_{u}:$ & Required force to initialize cutting fracture \\
\hline$P_{n}$ and $P_{t}$ : & $\begin{array}{l}\text { Normal and tangential component of total } \\
\text { applied external force, respectively }\end{array}$ \\
\hline${ }_{n u}$ and $P_{t u}$ : & $\begin{array}{l}\text { Normal and tangential component of total } \\
\text { cutting force during fracture, respectively }\end{array}$ \\
\hline and $P$ & $\begin{array}{l}\text { Normal and tangential component of a poin } \\
\text { applied external force, respectively }\end{array}$ \\
\hline$q_{n}$ and $q_{t}$ : & $\begin{array}{l}\text { Maximum of the area force intensity } \\
\text { between }[-w, w] \text { in the normal and } \\
\text { tangential direction, respectively }\end{array}$ \\
\hline : & $\begin{array}{l}\text { Distance between point } o \text { and point } B \text { (see } \\
\text { Figure } 3(\mathrm{~b}) \text { ) }\end{array}$ \\
\hline$R:$ & etween point $A$ and point $B$ (see \\
\hline$w:$ & $\begin{array}{l}\text { he top side of the trapezoid profile } \\
\text { ensity distribution (see Figure 5) }\end{array}$ \\
\hline$x:$ & $\begin{array}{l}\text { Slicing angel, that is, the angle between } P \\
\left.\text { and } P_{n} \text { (see Figures } 3(\mathrm{~b}) \text { and } 2\right)\end{array}$ \\
\hline and $\tau$ : & $\begin{array}{l}\sigma \text { is the tensile stress or just an expression of } \\
\text { any of the stress components and } \tau \text { is only } \\
\text { the shear stress }\end{array}$ \\
\hline$\tau_{i j}:$ & Maximum shear stress, $i$ or $j=1,2$, or 3 \\
\hline$\tau_{\max }:$ & Maximum of the maximum shear stresses \\
\hline 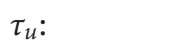 & ir stress \\
\hline$\mu:$ & \\
\hline$\eta:$ & \\
\hline $70:$ & $\begin{array}{l}\text { Relative sharpness factor of the sharpest } \\
\text { blade }\end{array}$ \\
\hline & hess factor of the dullest bl \\
\hline & arpness level \\
\hline
\end{tabular}

\section{References}

[1] K. Hellan, Introduction to Fracture Mechanics, McGraw-Hill, New York, NY, USA, 1984.

[2] M. Mahvash and Hayward V., "Haptic rendering of cutting: a fracture mechanics approach," Haptics-e, vol. 2, no. 3, pp. 112, 2001.

[3] A. G. Atkins, X. Xu, and G. Jeronimidis, "Cutting, by 'pressing and slicing,' of thin floppy slices of materials illustrated by experiments on cheddar cheese and salami," Journal of Materials Science, vol. 39, no. 8, pp. 2761-2766, 2004.

[4] A. Atkins and Y. Mai, Elastic and Plastic Fracture: Metals, Polymers, Ceramics, Composites, Biological Materials, Ellis Horwood, Chichester, UK, 1985.

[5] I. Kamyab, S. Chakrabarti, and J. G. Williams, "Cutting cheese with wire," Journal of Materials Science, vol. 33, no. 11, pp. 2763-2770, 1998.

[6] S. Way, "Some observations on the theory of contact pressures," Journal of Applied Mechanics, vol. 7, pp. 147-157, 1940.

[7] M. Sadd, Elasticity: Theory, Applications, and Numerics, Elsevier, Burlington, Mass, USA, 2005.

[8] Z. Xu, Elasticity Mechanics, Higher-level Education, 5th edition, 1996.

[9] A. E. H. Love, "The stress produced in a semi-infinite solid by pressure on part of the boundary," Philosophical Transactions of the Royal Society A, vol. 667, pp. 377-420, 1929.
[10] J. Boussinesq, Application des Potentiels a I'Etude de l'Euqilibre et du Mouvement des Solides Elastiques, Gauthier-Villars, Paris, France, 1885.

[11] F. F. Ling, M. Lai, and D. Lucc, Fundamentals of Surface Mechanics: with Applications, John Wiley Sons, Nwe York, NY, USA, 1973.

[12] S. Timoshenko, Theory of Elasticity, McGraw-Hill, New York, NY, USA, 3rd edition, 1970.

[13] K. L. Johnson, Contact Mechanics, Cambridge University, New York, NY, USA, 1985.

[14] A. E. H. Love, A Treatise on the Mathematical Theory of Elasticity, Cambridge University, New York, NY, USA, 4th edition, 1927.

[15] J. R. Dydo and H. R. Busby, "Elasticity solutions for constant and linearly varying loads applied to a rectangular surface patch on the elastic half-space," Journal of Elasticity, vol. 38, no. 2, pp. 153-163, 1995.

[16] O. J. Svec and G. M.L. Gladwell, "An explicit boussinesq solution for a polynomial distribution of pressure over a triangular region," Journal of Elasticity, vol. 1, no. 2, pp. 167-170, 1971.

[17] J. Li and E. J. Berger, "A Boussinesq-Cerruti solution set for constant and linear distribution of normal and tangential load over a triangular area," Journal of Elasticity, vol. 63, no. 2, pp. 137-151, 2001.

[18] G. M. Hamilton and L. E. Goodman, "The Stress field created by a circular sliding contact," Journal of Applied Mechanics, vol. 33, pp. 371-376, 1966.

[19] A. C. Fischer-Cripps, Introduction to Contact Mechanics, Springer, New York, NY, USA, 2000.

[20] H. Yoshihara and A. Matsumoto, "Measurement of the shearing properties of wood by in-plane shear test using a thin specimen," Journal of International Academy of Wood Science, vol. 39, no. 2, pp. 141-152, 2005.

[21] P. Lucas, Dental Functional Morphology: How Teeth Work, Cambridge University Press, New York, NY, USA, 1st edition, 2007.

[22] Tech Edge 2005, http://www.furitechnics.com.au/information/Tech\%20Edge/Cutting\%20edge\%20angles.htm.

[23] R. W. McGorry, P. C. Dowd, and P. G. Dempsey, "The effect of blade finish and blade edge angle on forces used in meat cutting operations," Applied Ergonomics, vol. 36, no. 1, pp. 71$77,2005$.

[24] R. L. Szabo, R. G. Radwin, and C. J. Henderson, "The influence of knife dullness on poultry processing operator exertions and the effectiveness of periodic knife steeling," American Industrial Hygiene Association Journal, vol. 62, no. 4, pp. 428433, 2001.

[25] D. Zhou and G. McMurray, "Modeling of blade sharpness and compression cut of biomaterials," Robotica, vol. 28, no. 2, pp. 311-319, 2010.

[26] C. Lipson and R. Juvinall, Handbook of Stress and Strength: Design and Material Applications, Macmillan, New York, NY, USA, 1963.

[27] ABB Robotics, "Product Manual IRB 140," ABB Robotics Products AB publication, no. M2000, article 3HAC 7564-1, 2000.

[28] ATI Industry Automation, "Installation and operations manual for ISA F/T-16," ATI Industry Automation Inc., 1998, http://www.ati-ia.com/app_content/documents/9610-051019\%20ISA\%20FT-16\%20\%28ALL\%29.pdf. 

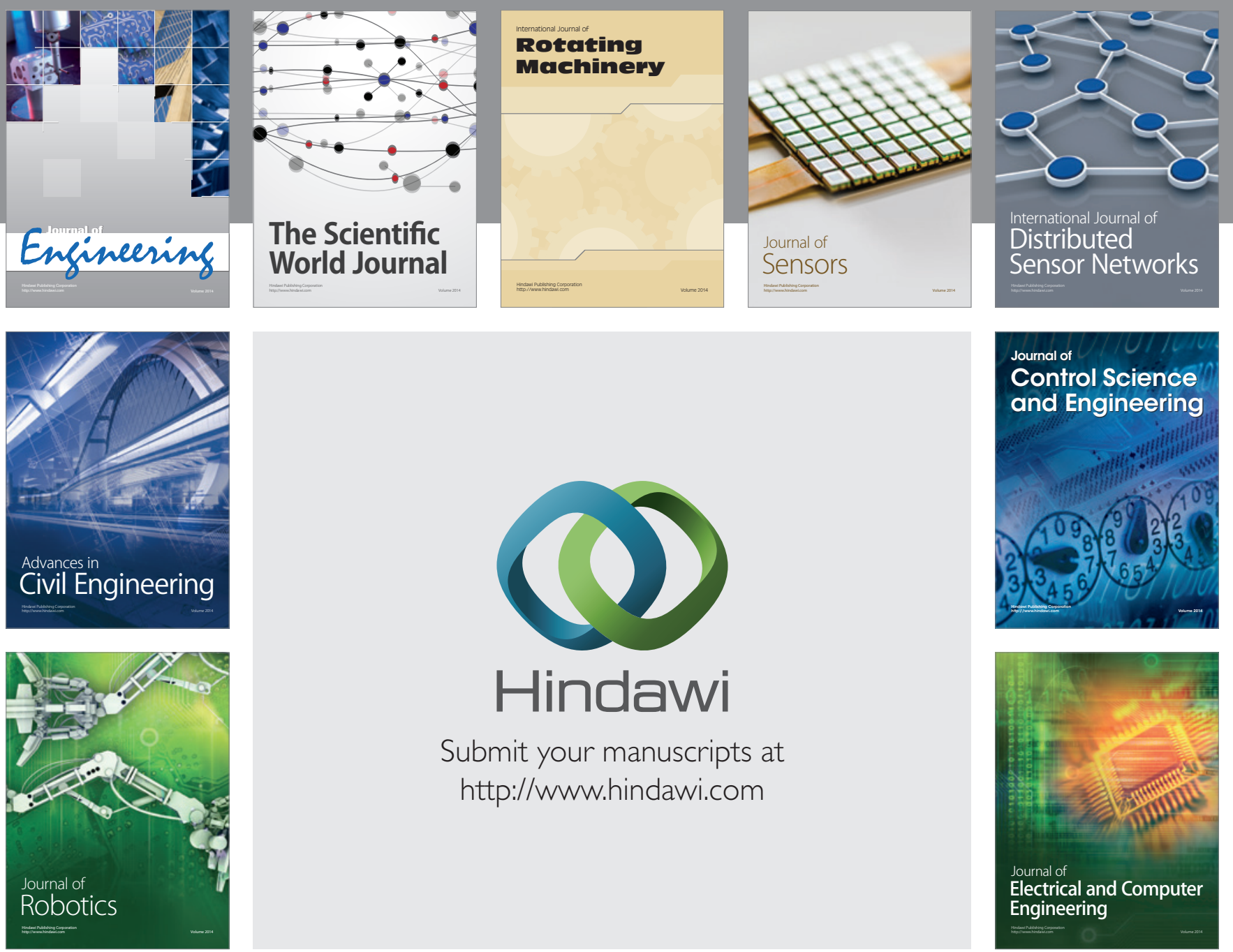

Submit your manuscripts at

http://www.hindawi.com
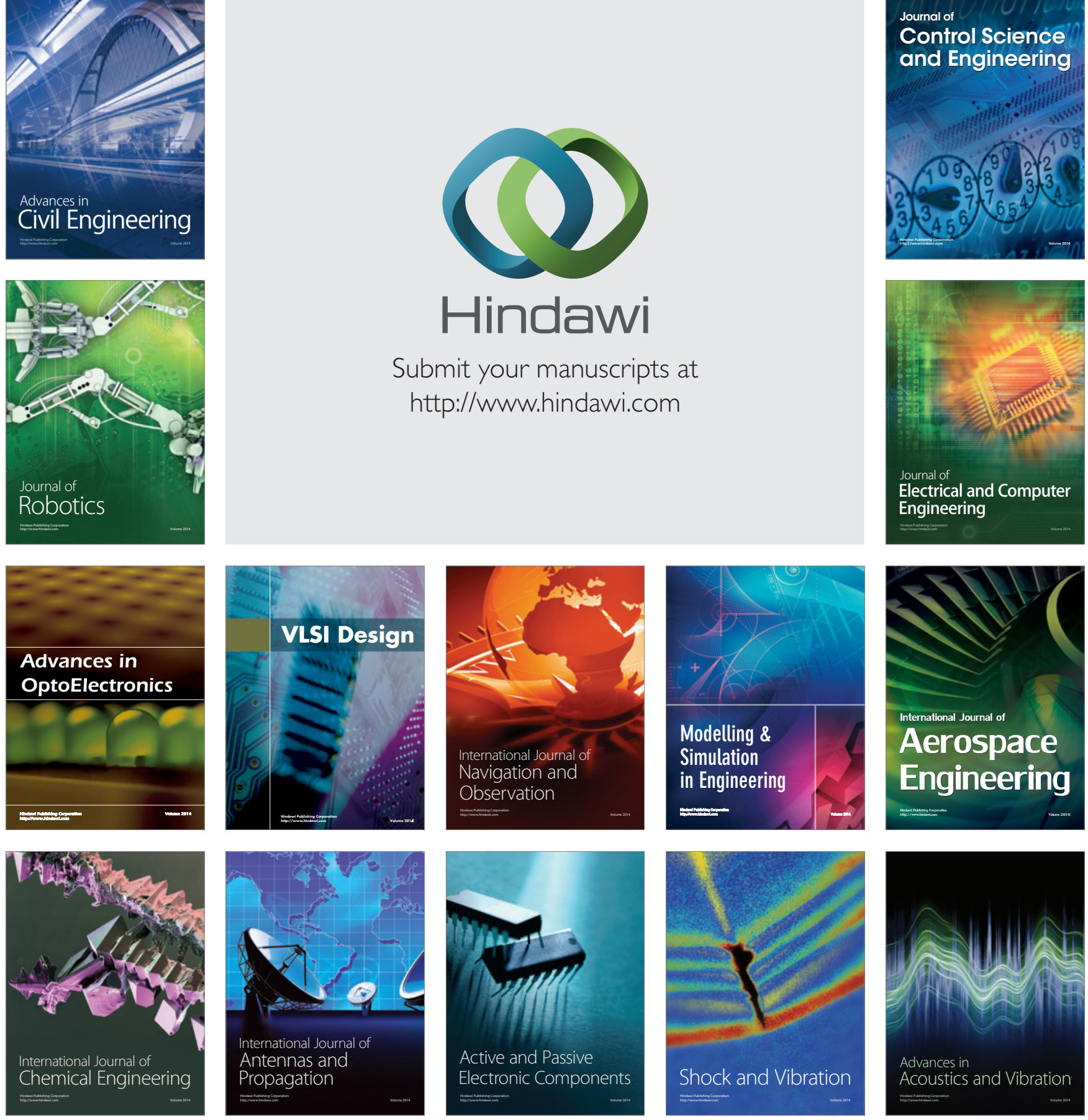